\title{
Characteristics of Flameless Combustion in 3D Highly Porous Reactors under Diesel Injection Conditions
}

\author{
M. Weclas ${ }^{1}$ and J. Cypris ${ }^{2}$ \\ ${ }^{1}$ Georg Simon Ohm University of Applied Sciences Nuremberg, Department of Mechanical Engineering, \\ Kesslerplatz 12, 90489 Nuremberg, Germany \\ ${ }^{2}$ Fraunhofer Institute for Building Physics IBP, Department of Energy Systems, Nobelstrasse 12, 70569 Stuttgart, Germany \\ Correspondence should be addressed to M. Weclas; miroslaw.weclas@ohm-hochschule.de
}

Received 18 January 2013; Accepted 19 February 2013

Academic Editor: Eliseo Ranzi

Copyright (C) 2013 M. Weclas and J. Cypris. This is an open access article distributed under the Creative Commons Attribution License, which permits unrestricted use, distribution, and reproduction in any medium, provided the original work is properly cited.

\begin{abstract}
The heat release process in a free volume combustion chamber and in porous reactors has been analyzed under Diesel engine-like conditions. The process has been investigated in a wide range of initial pressures and temperatures simulating engine conditions at the moment when fuel injection starts. The resulting pressure history in both porous reactors and in free volumes significantly depends on the initial pressure and temperature. At lower initial temperatures, the process in porous reactors is accelerated. Combustion in a porous reactor is characterized by heat accumulation in the solid phase of the porous structure and results in reduced pressure peaks and lowered combustion temperature. This depends on reactor heat capacity, pore density, specific surface area, pore structure, and heat transport properties. Characteristic modes of a heat release process in a two-dimensional field of initial pressure and temperature have been selected. There are three characteristic regions represented by a single- and multistep oxidation process (with two or three slopes in the reaction curve) and characteristic delay time distribution has been selected in five characteristic ranges. There is a clear qualitative similarity of characteristic modes of the heat release process in a free volume and in porous reactors. A quantitative influence of porous reactor features (heat capacity, pore density, pore structure, specific surface area, and fuel distribution in the reactor volume) has been clearly indicated.
\end{abstract}

\section{Introduction}

Future internal combustion engines are to feature a clean combustion process. Clean process means a homogeneous combustion requiring simultaneous (volumetric) ignition of a homogeneous (preferably premixed) charge. Such a process results in simultaneous heat release characterized by a homogeneous temperature field in the combustion chamber, and the process is flameless. In the literature, such a process in a free volume combustion chamber is often called HCCI. There are a number of challenges in realizing homogeneous combustion in an engine operating under variable load and speed conditions. Especially critical are control of ignition timing, combustion duration, heat release rate, and corresponding pressure gradient and pressure peak, control of combustion temperature for nearly zero- $\mathrm{NO}_{x}$ emissions, and completeness of the process for low $\mathrm{CO}$ and
HC emissions. There is no system known to the authors that can satisfy all conditions selected above, at least if variable load conditions are considered. Two of them receive special attention from the point of view of this paper's topic: control of ignition timing under variable air-excess ratios and lowering of combustion temperature below the thermal $\mathrm{NO}_{x}$-level. A novel kind of engine with a combustion process in highly porous three-dimensional reactors that could satisfy the above conditions has been proposed in [1]. This engine concept has great potential for high cycle efficiency and for a nearly-zero emission level allowing combustion temperature control below thermal $\mathrm{NO}_{x}$-formation. This, however, requires that all engine processes related to mixture formation, ignition, and combustion must perform in porous reactor volume, only. The nature and the character of processes, such as fuel injection, mixture homogenization, heat release, and thermodynamic of the cycle, are significantly 
different from the conditions known for free volume combustion chamber. Unfortunately, this kind of combustion process has almost never been investigated experimentally before under engine cycle conditions. Very specific features of porous combustion reactor significantly influencing mixture preparation and combustion conditions, not relevant to free volume combustion chamber, are porosity, heat capacity, and structure of the reactor. This kind of combustion system has a great potential for simultaneous realization of high cycle efficiency (heat recuperation during the cycle), elimination of soot emissions, and lowering of combustion temperature (reactor temperature) below thermal $\mathrm{NO}_{x}$-level. One of the crucial features of this kind of combustion technology is heat transfer processes between the solid and the fluid phase inside the porous medium simultaneously to heat release process and heat accumulation in reactor structure. This effect is clearly recognizable in decreased pressure peaks and lower pressure gradients, as compared to free volume combustion chamber. Owing to these significant different thermodynamic conditions, one of the critical questions concerns timescale and the rate of oxidation processes in porous reactors and their applicability to internal combustion engine cycle conditions. These aspects are discussed in the present paper, and the attention is paid to particular processes of mixture formation and combustion realized in a porous reactor volume. Especially in this case, there is a lack of information on the nature of real processes performed in porous reactors under engine-like conditions. Additionally, development of a high-temperature open cell and highly porous structures for application to internal combustion engines is necessary for development of this kind of combustion systems [2]. Processes performed inside porous reactor can be divided into two groups: direct fuel injection into a porous reactor and low- and high-temperature oxidation in the reactor. The former processes include Diesel-jet interaction with the highly porous structure described with reference to multijet splitting and fuel vaporization in a hot reactor [36]. Low-temperature oxidation processes include cool- and blue-flame reactions that occur just after injection begins during the ignition delay time period $[7,8]$. Thermal ignition and high-temperature oxidation (heat release) complete the investigated process [9-12]. Because of scarcity of experimental data in the literature, the present paper presents selected aspects of these complex phenomena as performed in porous reactors under engine-like conditions. A heat release process in porous reactors having different structures and heat capacities is discussed in comparison with a Diesellike process (free volume combustion). In both cases, a direct fuel injection using a common-rail diesel injection system into the combustion chamber (free volume or porous reactor) is used. Heat release process in a free volume combustion chamber has been described in [7]. The present paper extends this investigation on the process performed inside highly porous three-dimensional open cell combustion reactors.

The focus of the present paper is to investigate the basic process behavior not only for typical engine variable conditions such as pressure and temperature at the moment of fuel injection, but also for different reactor structures and architectures. This is also clear to the authors that it is still impossible to quantify the process data in detail with porous reactor structure, its porosity, heat capacity, specific surface area, and so forth. Along the direct fuel injection into reactor volume, spray interaction with structure wall junctions and heat transfer from the reactor to fuel for its vaporization are very complex and very sensitive to any changes in reactor parameters and remain still not well recognized or understood [13].

\section{Characterization of Highly Porous Reactors}

There are two main groups of different applications of porous materials and structures to internal combustion engines: porous structures applied to exhaust posttreatment systems for reduction of engine emissions (outside the engine cylinder and combustion chamber), see, for example, [14, 15]; porous structures applied to engine processes, especially inside the engine cylinder (combustion chamber) $[2,13]$. In the latter case, heat recuperation in a solid phase of the porous structure performs automatically, as the structure has contact with hot burned gases. Different aspects of heat recuperation in a porous structure applied to thermal engines are described in [16-25]. From the point of view of the application of porous reactors to in-cylinder engine processes (e.g., fuel distribution and vaporization, mixing, ignition, combustion, and heat recuperation), there are a number of requirements on structures and material properties [13]. Generally, the most important parameters of PM-structures as applied to engine processes, and especially to the combustion process, can be specified as follows.

(i) High porosity: more than $80 \%$ porosity with open pores (cells) for gas or liquid flow. Different processes in PM volume require different pore size (e.g., requirements for fuel distribution in space, for heat recuperation, and for combustion process are different). Direct influence on pressure losses. Development of nonfoam structures, for example, 3D macrocellular structure [3], the pore size, and its distribution in space may be optimized for particular application.

(ii) Large heat capacity must be adapted to particular application. In case of engine combustion in a porous reactor, it defines the dynamic properties of the engine and influences cycle thermodynamics and cold-start conditions.

(iii) Large specific surface area must be adapted to each particular application for supporting individual processes, especially for interphase heat transfer influencing the thermodynamics of the engine cycle.

(iv) Maximum temperature depends on the application, for example, for combustion in a porous reactor $T_{\text {max }}<2000 \mathrm{~K}$.

(v) Further aspects: thermal shock resistance; corrosion resistance; mechanical stability of the porous medium, material surface properties; electrical properties (for electrical heating of PM structures); longtime stability; 
Reactors made of $\mathrm{SiC}$ foam structures having different pore densities and reactor of high density wire packing used in the present investigation are shown in Figure 1. Specification of investigated reactors is given in Table 1.

\section{Combustion Chamber, Process Model, and Test Conditions}

3.1. Combustion Chamber. For experimental investigation of low- and high-temperature oxidation processes in porous reactors under engine-like conditions with wide flexibility in setting the test conditions, a special high-pressure, hightemperature, constant volume, and adiabatic combustion chamber has been built and equipped with a Diesel commonrail injection system (a standard Diesel oil is used), as already described in [7] - see Figure 2. The system simulates the thermodynamic conditions at the time instance corresponding to the nearly TDC of compression in a real engine equipped with porous reactor [1]. Initial gas pressure (corresponding to the time instant when fuel injection starts) and reactor temperature at this moment can be chosen independently of one another. The reactor is heated electrically, and it is assumed that at the moment of fuel injection start, the gas trapped in the reactor has reactor temperature. The injection and heat release processes have been proved on their repeatability for presented analyses. The pressure in the chamber is measured with a piezoelectric pressure transducer which is able to switch between static pressure measurement and highly dynamic pressure change measurement. A synthetic dry air is used as combustion air and is supplied with variable pressure. The temperature inside the chamber is measured with a thermocouple, and static conditions are preset prior to the start of experiment. More information is available in [7].

3.2. Free Volume Reactor and Porous Reactor Models. In the case of a free volume reactor model, the combustion chamber is considered as a closed system having constant volume. According to the first law of thermodynamics for a constant volume and an adiabatic system, the changes in the internal energy directly correspond to the heat release rate in the chamber. In the case of our porous reactor model, part of the heat released during the investigated process is accumulated in the solid phase of the reactor, which has much higher heat capacity as the gas trapped inside. This fact may significantly influence the pressure history, as explained in Figure 3. This figure shows a model of an adiabatic free volume reactor (left hand side in Figure 3) and an adiabatic highly-porous reactor (right hand side in Figure 3). The adiabatic free volume reactor consists of a constant volume adiabatic combustion chamber filled with working gas. During this virtual experiment, a given amount of energy $E_{\mathrm{ch}}$ is supplied with the injected fuel $\left(H_{u} \times m_{\text {fuel }}\right)$ into the chamber. As a result of oxidation processes (heat release), this energy is converted into heat represented by $Q_{\text {in }}$ and in consequence increases the internal energy of the gas $\Delta U_{\mathrm{GAS}(\mathrm{FV})}$ and corresponding temperature changes $\Delta T_{\mathrm{GAS}(\mathrm{FV})}$. In a constant volume and adiabatic combustion
TABLE 1: Characteristics of investigated porous reactors.

\begin{tabular}{llcccc}
\hline $\begin{array}{l}\text { Reactor } \\
\text { no. }\end{array}$ & Structure & $\begin{array}{c}\text { Pore } \\
\text { density } \\
(\mathrm{ppi})\end{array}$ & $\begin{array}{c}\text { Mean pore } \\
\text { size } \\
(\mathrm{mm})\end{array}$ & $\begin{array}{c}\text { Reactor } \\
\text { mass } \\
(\mathrm{g})\end{array}$ & $\begin{array}{c}\text { Porosity } \\
(\%)\end{array}$ \\
\hline 1 & Foam $(\mathrm{SiC})$ & 8 & 5.53 & 19.1 & 89.31 \\
2 & Foam $(\mathrm{SiC})$ & 10 & 4.99 & 22.2 & 89.56 \\
3 & Foam $(\mathrm{SiC})$ & 20 & 2.93 & 22 & 89.89 \\
4 & Foam $(\mathrm{SiC})$ & 30 & 2.67 & 21.6 & 90 \\
5 & Wire packing & $/$ & $/$ & 46.1 & ca. 90 \\
\hline
\end{tabular}

chamber, this temperature change is represented by measured pressure changes $\Delta p_{\mathrm{GAS}(\mathrm{FV})}$ within the chamber.

In the case of an adiabatic highly porous reactor, the system consists of a porous reactor (PM) and working gas trapped in the reactor volume. The system has a constant volume, and the combustion chamber is completely filled with the porous reactor. Similarly to a free volume chamber, the energy converted into heat $Q_{\text {in }}$ increases the internal energy of the gas trapped in the porous reactor $\left(\Delta U_{\mathrm{GAS}}\right.$ in PM$)$ and of the porous reactor itself $\left(\Delta U_{\mathrm{PM}}\right)$. Corresponding gas temperature change includes the energy increase of the porous reactor due to heat accumulated in PM [26]. Contrary to the free volume reactor, the gas trapped in a porous reactor volume cannot be thermally decoupled from the internal energy changes of the porous reactor. Additionally, it must be considered that the heat capacity of the porous reactor (represented by its mass) is many times higher $\left(10^{2}\right.$ to $\left.10^{3}\right)$ than the mass of gas trapped inside the PM volume. As a consequence, different changes in the gas temperature trapped in the PM volume result as compared to a free volume reactor. Finally, significantly reduced pressure changes $\left(\Delta p_{\mathrm{GAS}(\mathrm{PM})}\right)$ are measured as a result of the combustion process in the porous reactor as compared to the free gas adiabatic conditions $\left(\Delta p_{\mathrm{GAS}(\mathrm{FV})}\right)[26]$. In the present paper, both free volume and porous reactor systems have been investigated in a constant volume $\left(76 \mathrm{~cm}^{3}\right)$ combustion chamber. In one case, diesel fuel has been injected into free volume chamber containing hot air only (no air motion is applied). In another case, the fuel has been injected using the same diesel injector into the chamber completely filled with porous reactor.

3.3. Model of the Investigated Process. For analysis of the pressure history as measured right after fuel injection begins, a phenomenological model of a multistep oxidation (and ignition) [8] has been conceived and already used for analysis of Diesel-like (in free volume) combustion as described in [7]. This model has also been implemented to the process analysis in porous reactors, assuming that similar chemical reactions occur in both combustion systems. In this model, two measured parameters are investigated: pressure history and pressure gradient distribution, both as a function of time after injection begins. The whole process is analysed from time "zero" defined as a trigger signal for the Diesel injector (start of injection "IB"). The procedure for the setting of initial temperature $T_{\mathrm{IB}}=T_{\mathrm{PM}}$ and initial pressure $p_{\mathrm{IB}}$ in the combustion chamber filled with porous reactors is shown 


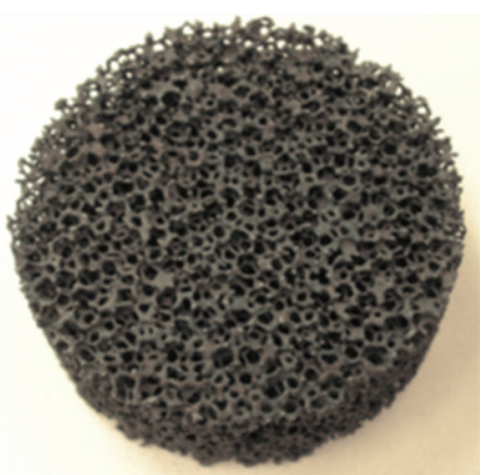

$\mathrm{SiC}$ foam (30 ppi)

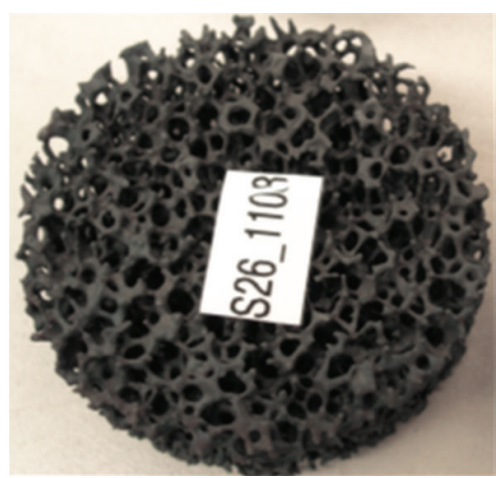

$\mathrm{SiC}$ foam (8 ppi)

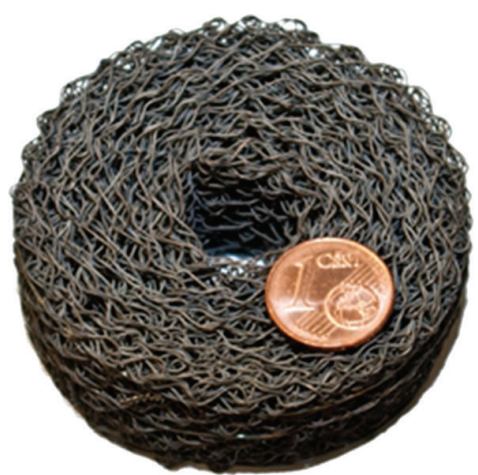

Wire packing

FIGURE 1: Examples of porous reactors used in the present investigation.

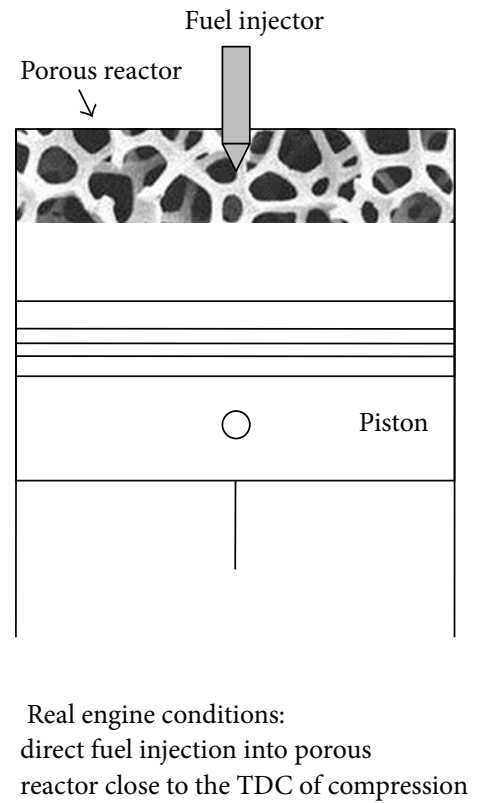

(a)

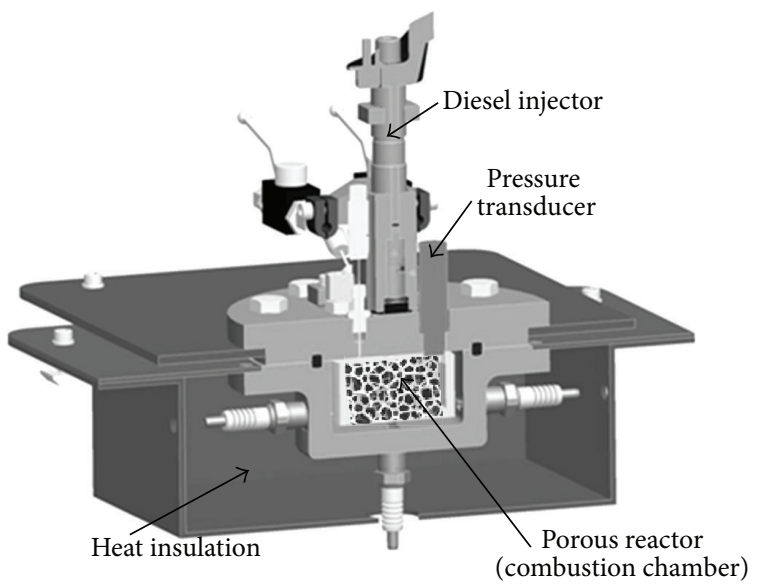

Real engine conditions: direct fuel injection into porous reactor under constant volume conditions

(b)

FIGURE 2: (a) The principle of real engine with combustion in a porous reactor; (b) engine simulator (combustion chamber) simulating engine conditions at TDC of compression.

in Figure 4. In a first step, a given mass of synthetic dry air at certain pressure $p_{1}$ is supplied to the chamber. After closing the system, the air is trapped in the porous reactor which is electrically heated up to the required temperature $T_{\mathrm{IB}}$ corresponding to the porous reactor temperature $T_{\mathrm{PM}}$. This also results in increasing chamber pressure to $p_{\mathrm{IB}}$. Characteristic time $t$ (delay time) of a particular phase of the process is analysed and measured, starting at zero-time point (point IB). For analysis of the reaction rate, a slope of the reaction curve corresponding to the particular oxidation process is described by average pressure changes in time [bar/ms] —-for details see [7].

3.4. Test Conditions Used in Present Investigation. There are different test conditions used in the present investigation. Most typical of them are measurements performed at constant parameters, for example, at constant initial temperature
$T_{\mathrm{IB}}$, at constant initial pressure $p_{\mathrm{IB}}$, for a constant air excess ratio, and for a constant mass of injected fuel. Investigations have been performed in the range of initial temperature $T_{\mathrm{IB}}$ from $200^{\circ} \mathrm{C}$ to $700^{\circ} \mathrm{C}$ and for initial pressure $p_{\mathrm{IB}}$ from 1 bar to 20 bar. A constant injection pressure (600 bar) of a commonrail Diesel injection system equipped with a six-hole nozzle has been used. A single injection process has been performed with a fixed injection characteristic shape: injector opening, injection duration, and injector closing. The nozzle opening duration depends on the mass of fuel to be injected and was varying from $250 \mu$ s to $670 \mu$ s. The Diesel injector as well as pressure transducer was water cooled.

\section{Results and Discussion}

4.1. Short Characterization of the Fuel Injection Process into a Porous Reactor. In the case of direct fuel injection into porous 


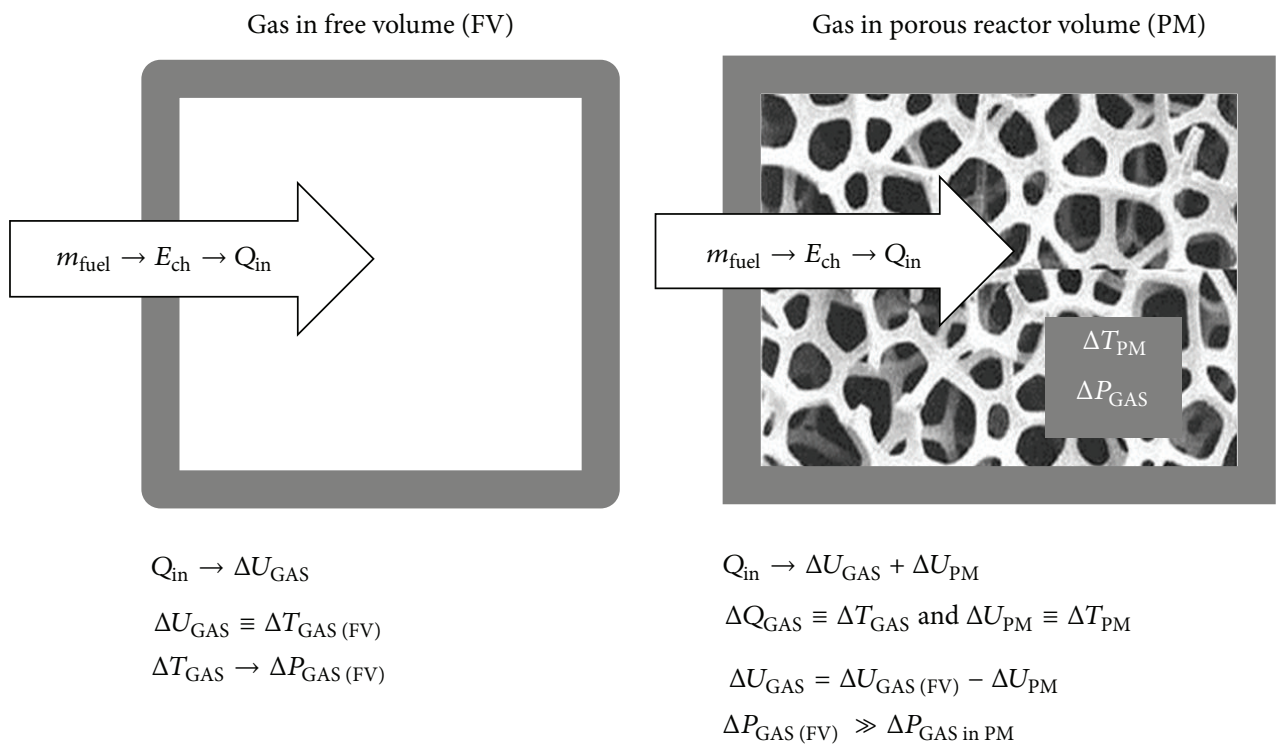

FIGURE 3: Comparison of thermodynamic conditions in adiabatic free volume system and in porous reactor.

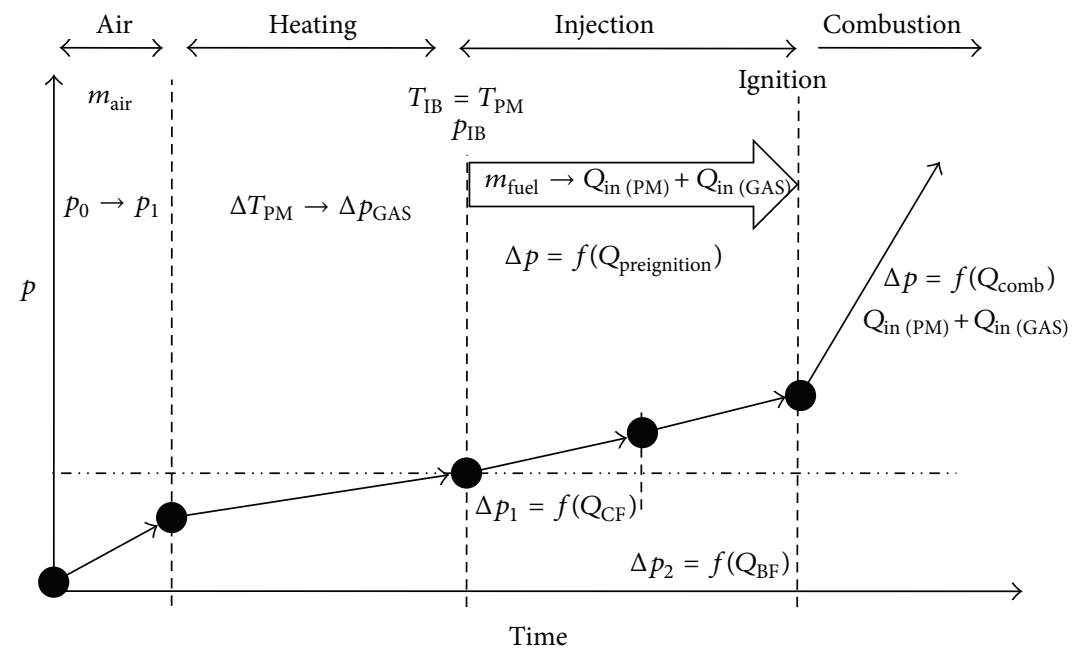

FIGURE 4: Routine of setting the test conditions (initial pressure and initial temperature) in free volume system and in porous reactor.

reactor, there is a need for understanding the fuel-jet interaction with porous structures and to find out the best conditions for mixture homogenization inside the reactor volume. This homogenization is required for a clean and homogeneous combustion process in a porous reactor. There is only limited information available on Diesel-jet interaction with porous structures [3-6]. Most important facts may be summarized as discussed below. The basic process of spray interaction with wall junctions of a three-dimensional porous structure is described by a multijet splitting effect-see Figure 5. Four characteristic phases of liquid-jet interaction with the (cold) porous medium have been selected [3-5]. Phase 1. The free jet penetrates throughout the available space between nozzle outlet and porous-medium surface. Phase 2. The jet impinges onto the PM surface. Phase 3. The jet propagates throughout (inside) the PM volume and interacts with wall junctions of the structure (multijet splitting). Phase 4. Part of the liquid may leave the PM volume (it depends on jet momentum, PM geometry, pore size and density). This effect can be utilized by fuel injection into a porous medium where the whole amount of fuel is trapped in the PM reactor volume. Injection into a hot reactor also permits a very quick fuel vaporization process with simultaneous mixing with combustion air [5].

\subsection{Heat Release Process in Porous Reactors and in a Free Vol-} ume Combustion Chamber. At the beginning of this section, a few examples of heat release as measured in porous reactors under Diesel engine-like conditions are presented in order to visualize the character of the analysed process. A heat release process as measured in an $\mathrm{SiC}$ foam reactor of low pore density (8 ppi) is presented and analysed in Figure 6. This figure shows pressure histories measured at three different initial reactor temperatures for different chamber pressures. The pictures on the left side represent the entire process measured, 


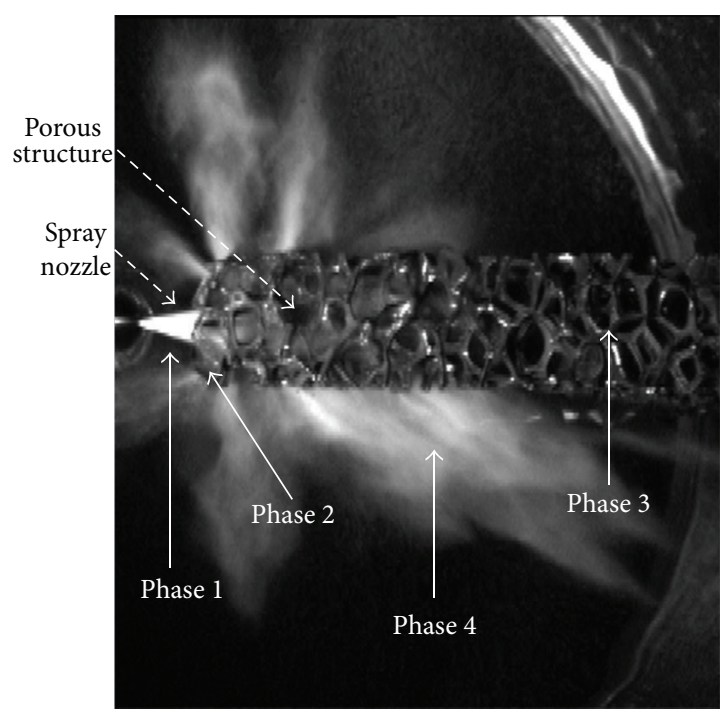

(a)
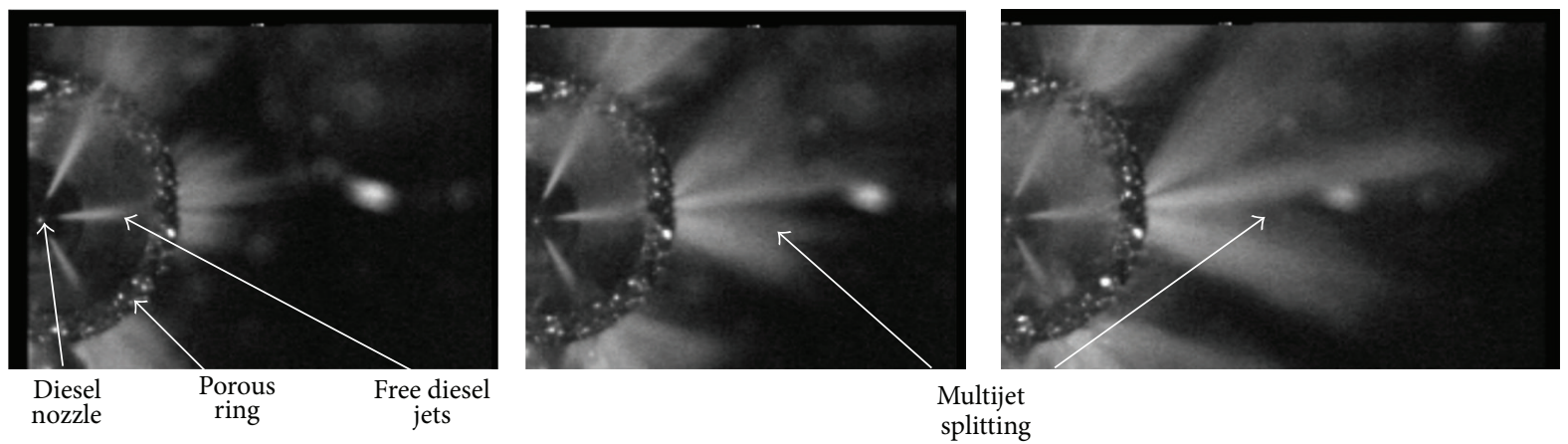

(b)

FIGURE 5: (a) Diesel spray interaction with highly porous structure; (b) example of a multijet splitting effect based on the interaction of Diesel spray with a porous structure (porous ring in the figure) at different time instances (at $t=0$ Diesel jet touches the inner surface of the porous ring).

and the right hand side pictures are plotted within the limited range of pressure change for better analysis of the initial part of the heat release process. The heat release process in a porous reactor consists of steps similar to those defined for free volume combustion [7]. Just after the fuel injection starts, the fuel partly vaporizes and the pressure changes to the negative range and is followed by low-temperature oxidation reactions: cool- and blue-flames being exothermic reactions resulting in a positive change of pressure. After this phase of the process, a high-temperature oxidation represented by a very quick pressure increase in the combustion chamber is observed. The presence of the porous reactor with its large heat capacity changes the thermodynamic conditions of the process. Generally, the higher the pressure in the chamber at the moment of fuel injection, the faster the heat release process, especially preignition reactions. In a very early stage of the process, fuel vaporization seems to be less dependent on the chamber pressure at lower temperature. With increasing reactor temperature, the process is much faster and more pressure dependent. The higher the initial chamber pressure, the shorter delay time and the faster heat release. The maximum of chamber pressure change is less dependent on initial temperature and should be correlated with heat accumulation in the porous reactor (see Section 3.2).

It will be useful now to compare this heat release process with conditions in a free volume combustion chamber (without porous reactor), as shown in Figure 7. This figure shows pressure histories (left) and pressure gradient distributions (right) at a constant initial reactor temperature $\left(400^{\circ} \mathrm{C}\right)$ at three different initial pressures $\left(p_{\mathrm{IB}}=8,12\right.$, and 18 bar). There are two main differences in the process observed in porous reactors and in the free volume. Firstly, a pressure peak of much greater value is recorded in free volume combustion. This is due to heat transfer to the porous reactor according to its large heat capacity as compared to heat capacity of gas trapped in the reactor volume (see model in Figure 3) [26]. Secondly, the delay time of the process is much shorter in the case of a porous reactor due to the very effective heat transfer inside the reactor volume and nearly constant reactor temperature conditions. This effect is more intense with increasing initial chamber pressure. Pressure gradient distributions confirm this observation: reaction rate increases with pressure and spacing between both processes also increases. On the other hand the character of the 


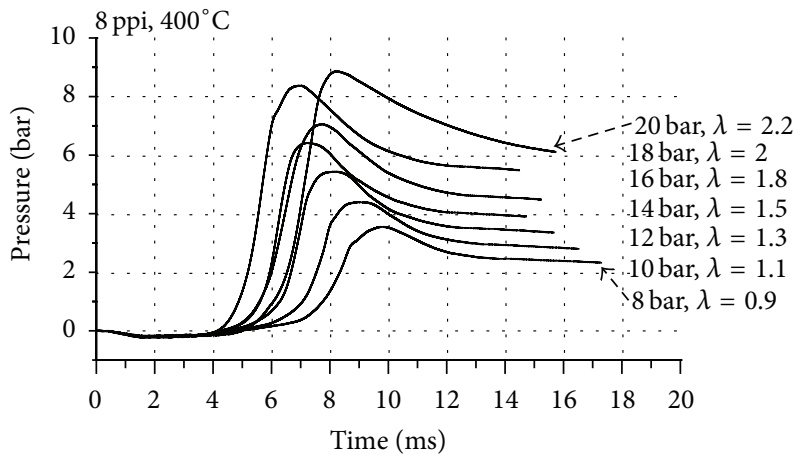

(a)

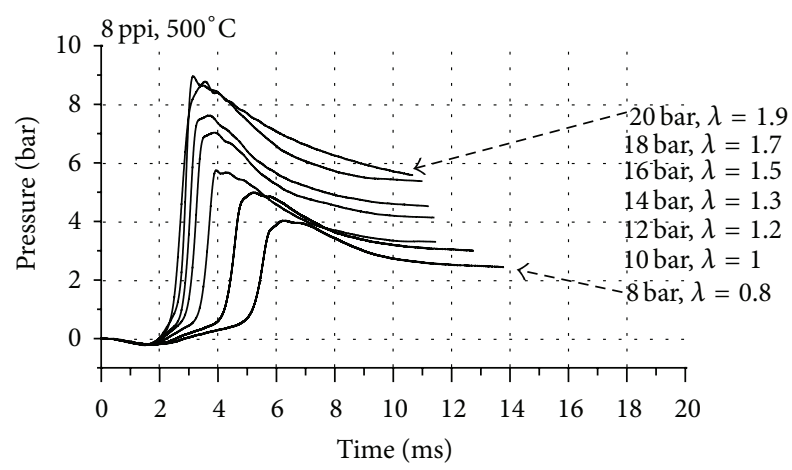

(c)

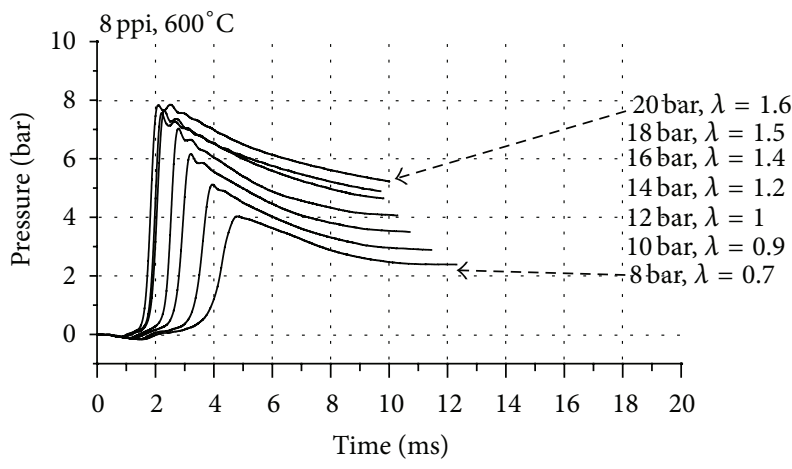

(e)

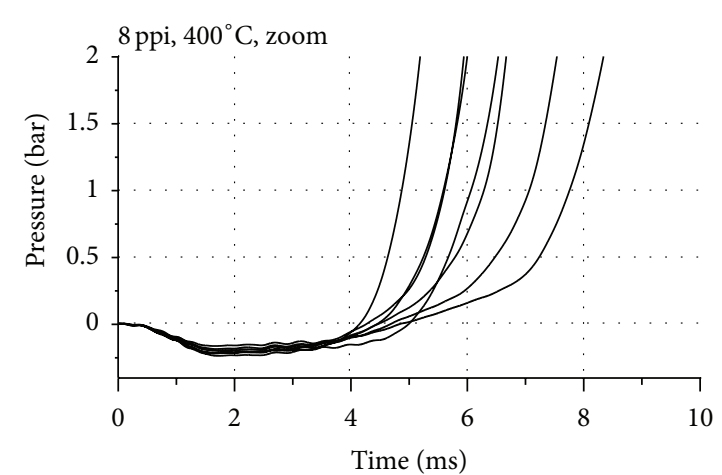

(b)

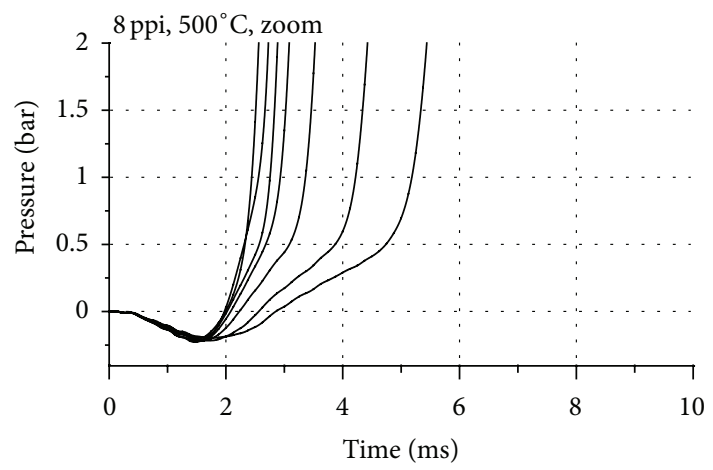

(d)

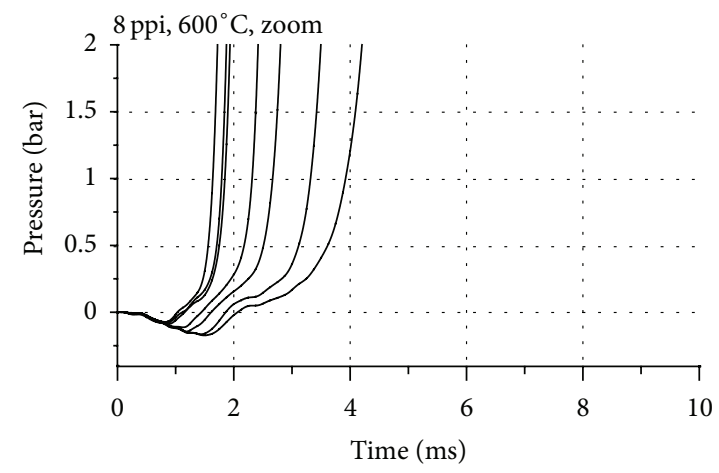

(f)

Figure 6: Pressure history for heat release in an $\mathrm{SiC}$ foam porous reactor (8 ppi) after fuel injection starts at three initial reactor temperatures $400^{\circ} \mathrm{C}, 500^{\circ} \mathrm{C}$, and $600^{\circ} \mathrm{C}$ for different initial pressures from 8 bar to 20 bar. The amount of injected fuel is $23.7 \mathrm{mg}$, and air excess ratio is not constant $\lambda=f(p)$; left-complete history; right-initial period of the process.

process seems to be similar for both free volume combustion and porous reactor combustion; however, the process in a porous reactor tends to be a multistep process. Pressure distribution changes its character from negative to positive much faster in a porous reactor and takes as little as $50 \%$ of the time for free Diesel conditions. Negative pressure change for free volume conditions is much higher than in a porous reactor due to the energy stored in the reactor. Vaporization enthalpy cannot change the temperature in the reactor so much as it has much more accumulated energy as is the case with gas in a free volume combustion chamber. The process in the same configuration is now investigated at different initial temperatures and at a constant initial pressure of 18 bar (Figure 8). This figure shows two kinds of information. The top plot represents an overview of the whole process and allows direct comparison of process dynamics at different initial temperatures. The bottom plots show details of the process at each investigated temperature separately, still comparing data for the porous reactor (solid lines) and for a free volume combustion chamber (dashed lines). Because of significant differences in the characteristic times corresponding to individual temperatures, the plots have different time scales.

At low initial temperatures, the heat release process is very much delayed (especially in a free volume) and significantly accelerates with increasing temperature. Generally, at each 


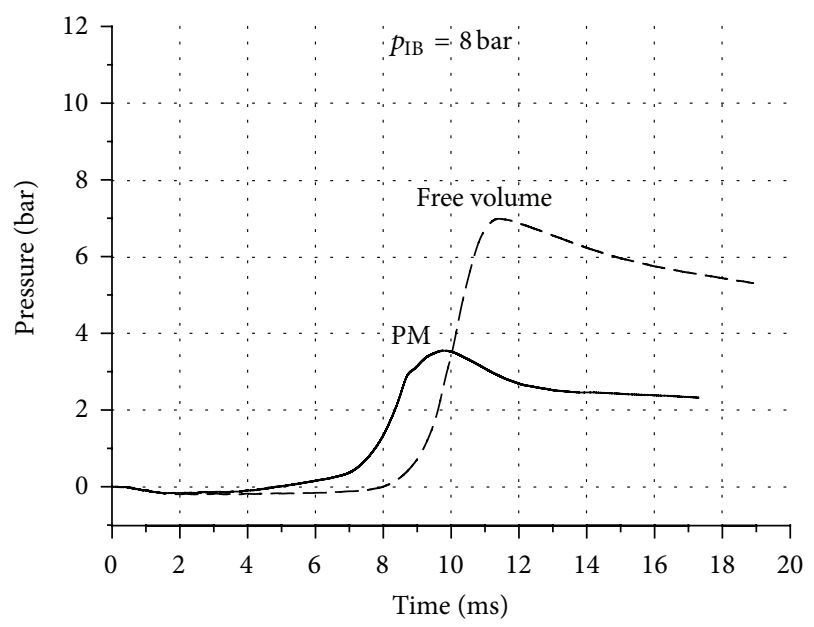

(a)

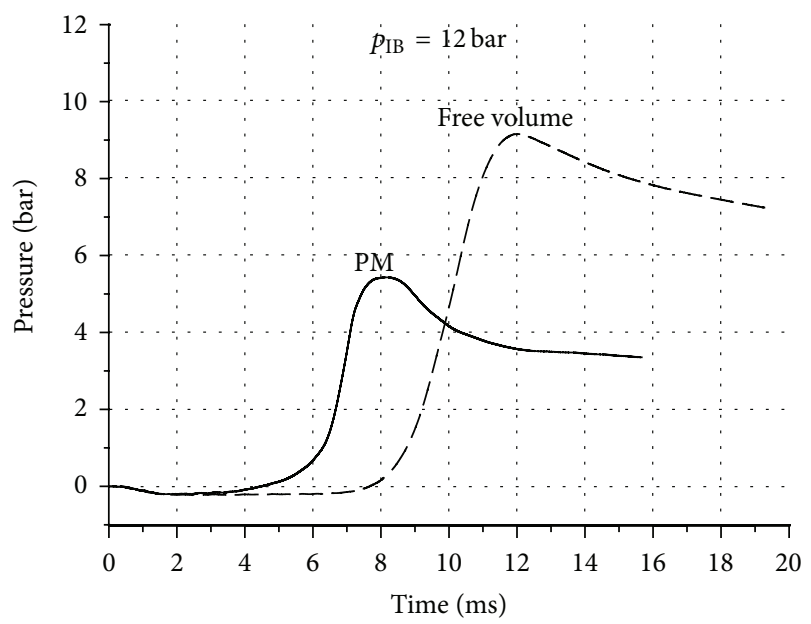

(c)

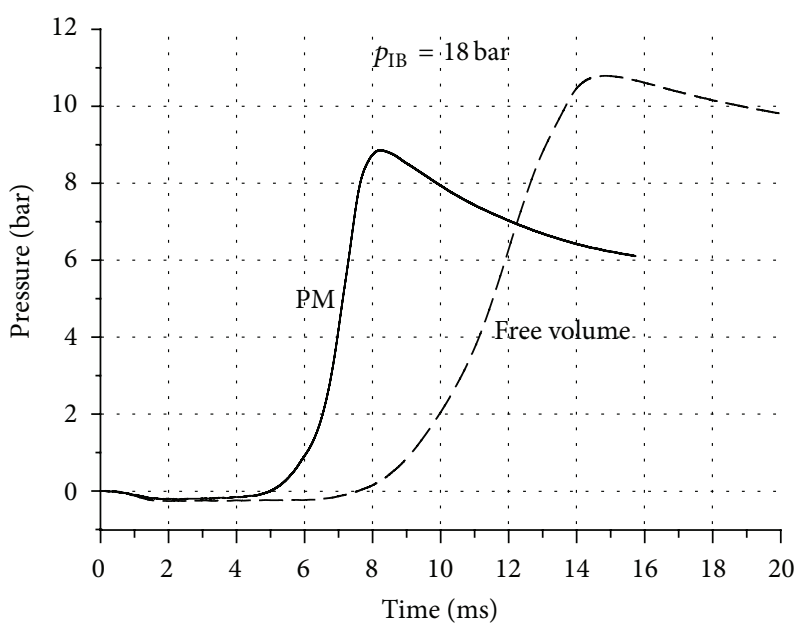

(e)

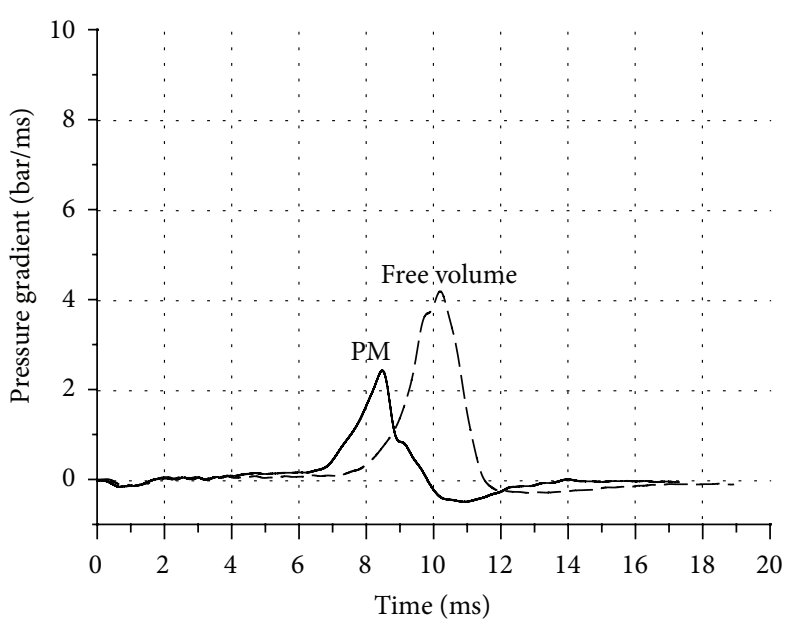

(b)

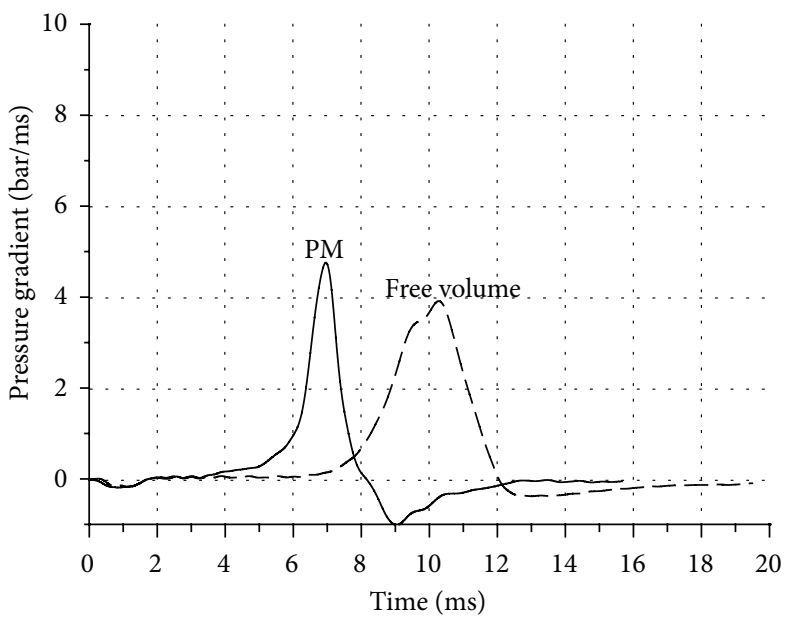

(d)

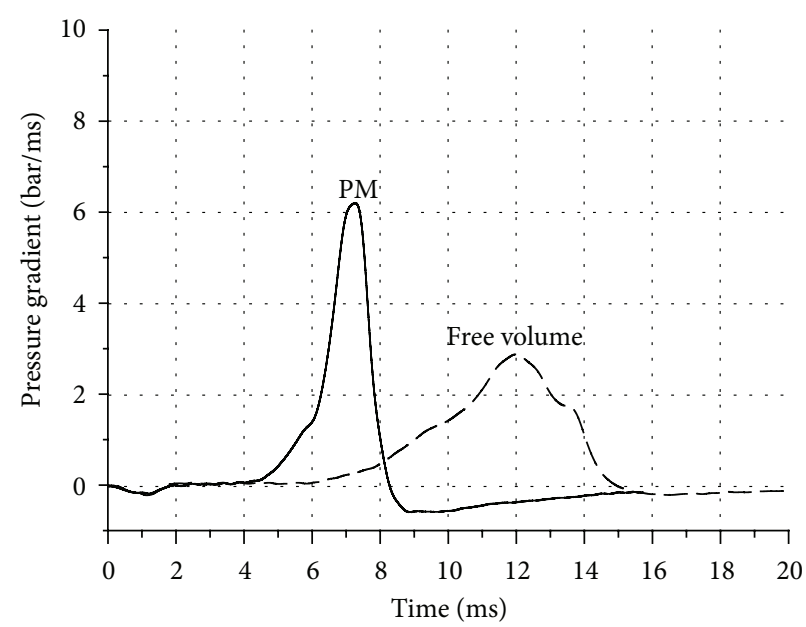

(f)

FIGURE 7: Comparison of pressure histories and pressure gradient distributions for heat release in free volume and in an SiC foam porous reactor ( $8 \mathrm{ppi}$ ) after fuel injection starts at three initial pressures and temperature $400^{\circ} \mathrm{C}$. The amount of injected fuel is $23.7 \mathrm{mg}$ : left-complete history; right-pressure gradients. 

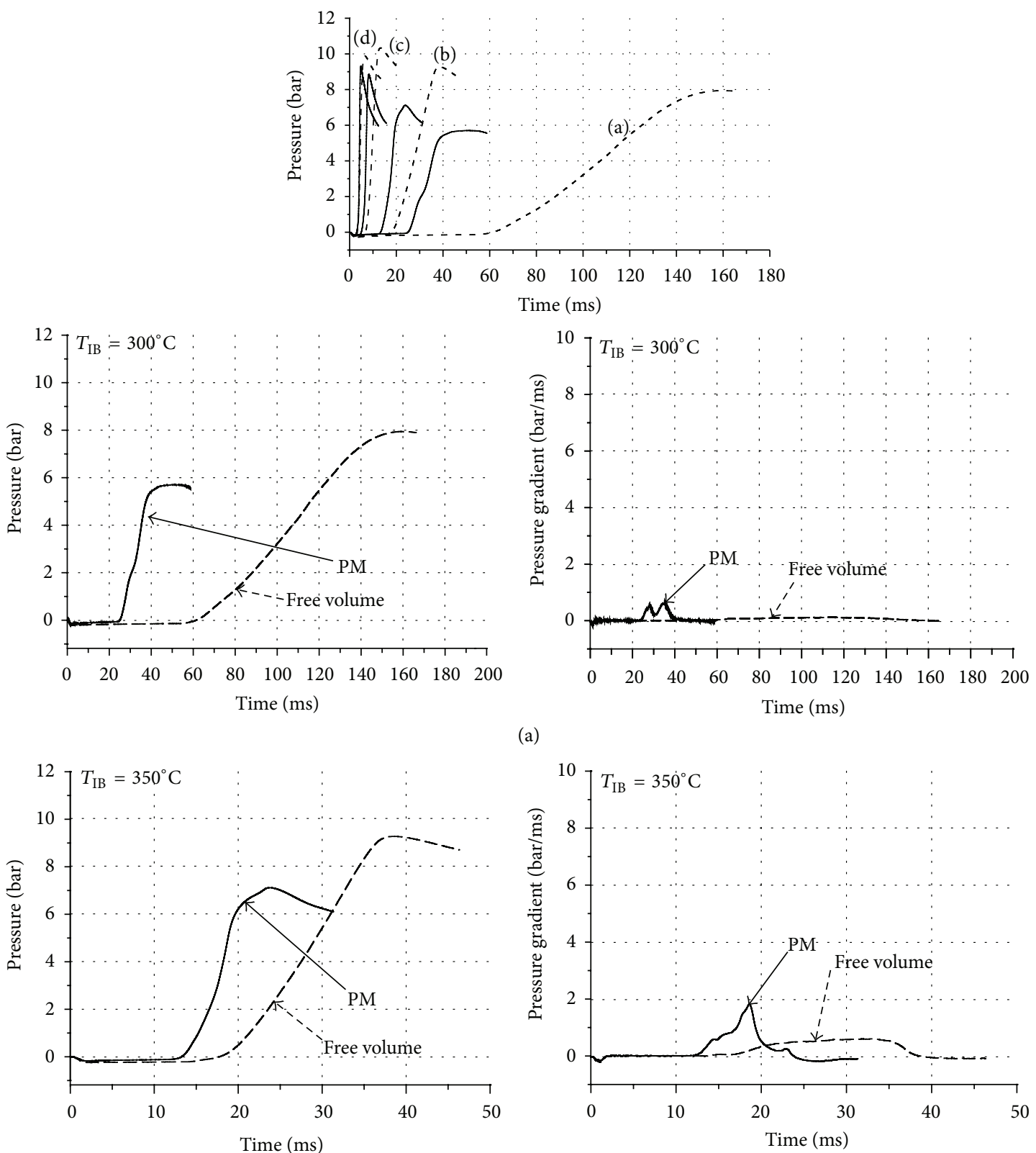

(b)
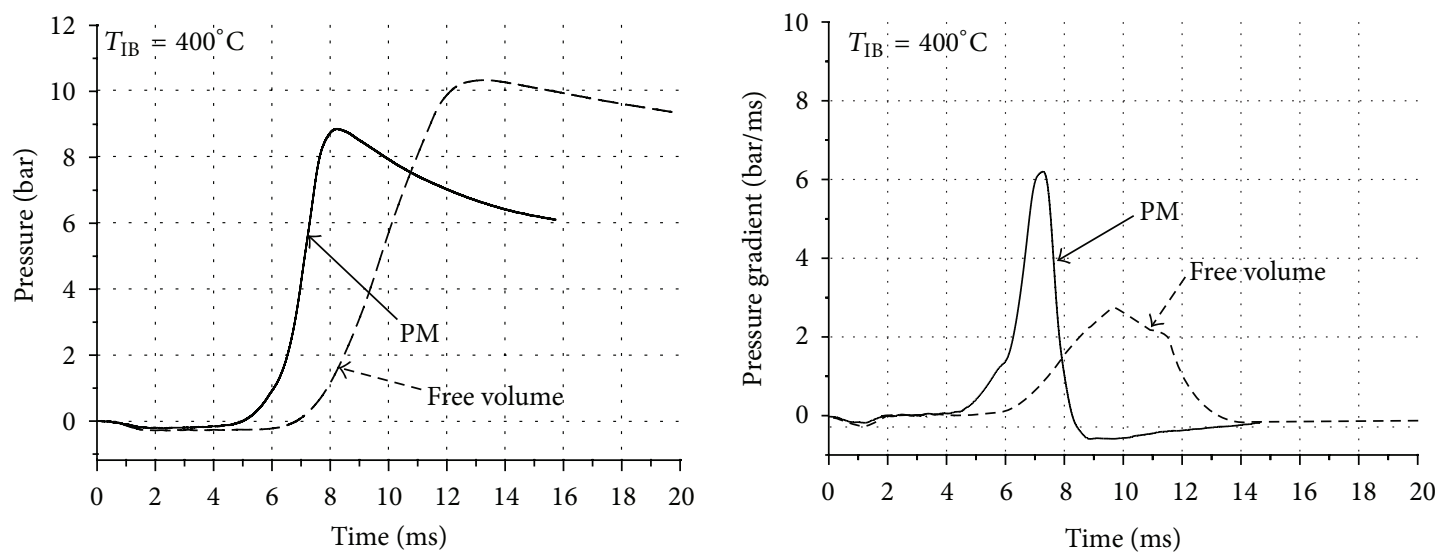

(c)

Figure 8: Continued. 

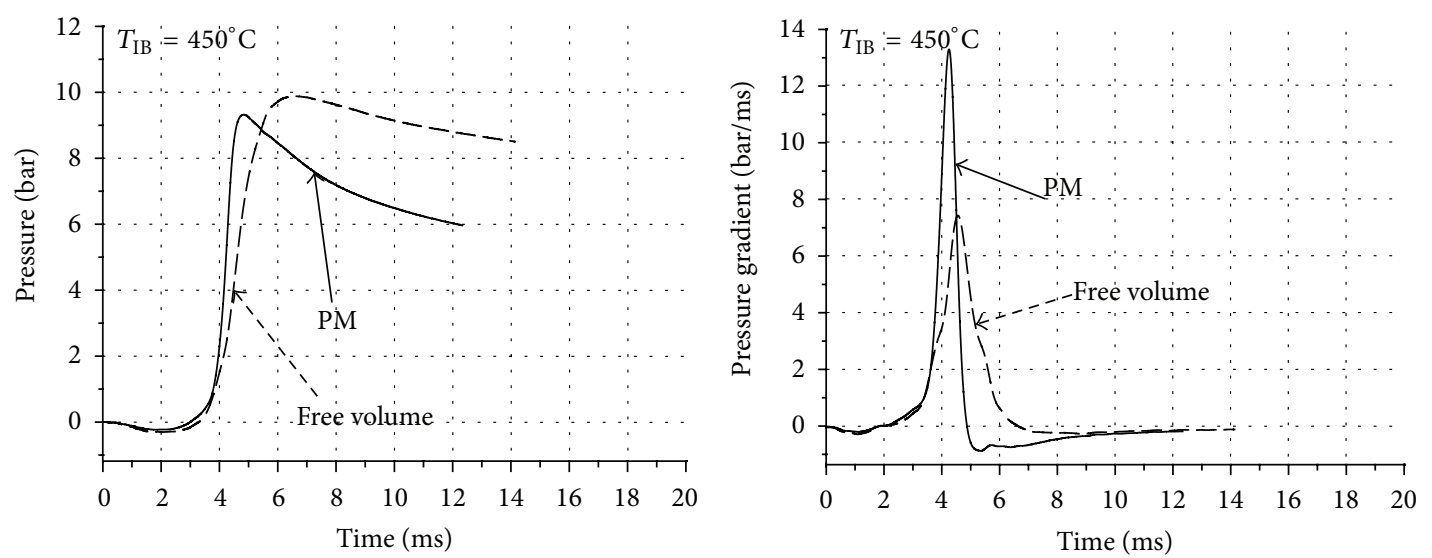

(d)

FIGURE 8: Comparison of pressure histories for heat release in free volume and in an $\mathrm{SiC}$ foam porous reactor (8 ppi) after fuel injection starts at different initial temperatures and at constant initial pressure $p_{\text {IB }}=18$ bar. The amount of injected fuel is $23.7 \mathrm{mg}$ : top-complete pressure history; (a) to (d) - process at individual temperature.

temperature the process delay in a porous reactor is shorter than in a free volume chamber, but the time spacing decreases with increasing initial temperature. The pressure history distribution shows the effect of heat accumulation in a porous reactor represented by reduced maximum pressure as compared to the free volume process. The pressure gradient distribution shows higher gradients of the heat release process in a porous reactor at all temperatures investigated. Nevertheless, the character of pressure and pressure gradient distributions seems to be similar for both conditions. This similarity will in detail be indicated in Section 4.4 where the characteristic modes of the heat release process in porous reactors and in a free volume chamber are analysed.

Next, the process in a high pore density reactor (30 ppi) is compared to free Diesel conditions (Figure 9). The process is analysed at three initial temperatures $\left(T_{\mathrm{IB}}=400^{\circ} \mathrm{C}, 500^{\circ} \mathrm{C}\right.$, and $\left.600^{\circ} \mathrm{C}\right)$ at three initial pressures $\left(p_{\mathrm{IB}}=10 \mathrm{bar}, 12\right.$, and 14 bar). Pressure histories are plotted at two different scales of pressure and time axis giving an overall view of the process (left) and of the initial part of heat release (right). As shown, for a high pore density reactor, the oxidation processes are accelerated and especially at the beginning of the process, the effect of reactor heat capacity is visible. This includes also differences in fuel distribution and vaporization inside the reactor as compared to a free volume combustion chamber. The process in the reactor is faster and is represented by less pressure change during the fuel vaporization process. Again the maximum chamber pressure is much less in the porous reactor owing to the reactor heat capacity and large specific surface area for interphase heat transfer. In general, under both test conditions the influence of initial pressure and temperature is similar. With increasing $p_{\mathrm{IB}}$, the maximum pressure during the heat release process increases. Furthermore, the delay times decrease with increasing initial pressure at initial temperatures of $500^{\circ} \mathrm{C}$ and $600^{\circ} \mathrm{C}$. In the case of free volume conditions, this effect is less visible at $T_{\mathrm{IB}}=400^{\circ} \mathrm{C}$. At higher initial temperatures $\left(500^{\circ} \mathrm{C}\right.$ and $600^{\circ} \mathrm{C}$ ), the character of the process under either conditions indicates a multistep ignition. At lower initial temperature
$T_{\mathrm{IB}}=400^{\circ} \mathrm{C}$, the heat release in a free volume is a single-step process contrary to a multistep process in a porous reactor.

\subsection{Comparison of the Heat Release Process in Different} Porous Reactors. Different foam reactors and other porous structures, like wire packings or macrocellular reactors [2], may significantly influence the heat release process. Reactors having different pore sizes and structures may in different manner influence the investigated process. There are three main groups of factors that have to be taken into consideration. The first group considers the influence of reactor heat capacity and is directly related to reactor mass and influenced by pore density. The second group considers heat capacity together with specific surface area and determines the heat exchange inside the reactor volume. The third group relates to pore size and pore structure of the reactor influencing the conditions for the fuel injection process and fuel distribution in the reactor volume.

An influence of pore density of foam reactors on the heat release process is depicted in Figure 10, which shows pressure history and pressure gradient distributions as measured for $p_{\mathrm{IB}}=10$ bar at two initial temperatures $\left(T_{\mathrm{IB}}=400^{\circ} \mathrm{C}\right.$ and $600^{\circ} \mathrm{C}$ ). The pressure peak level is pore density dependent and decreases with increasing reactor density for both investigated temperatures. In general, this behavior is related to the effect of heat capacity for heat accumulation in the reactor, of specific surface area influencing heat transfer in the reactor, and of pore size and its structure influencing spray distribution in reactor volume and corresponding mixture formation conditions. The complexity of this influence on the heat release process in porous reactors is indicated comparing pressure histories in low density reactors. The maximum pressure for reactors with 8 and 10 ppi pore density is similar at both investigated conditions.

Pressure gradient distributions show an influence of pore density on both the reaction rate level and the characteristic time. Generally, lower gradients are measured for higher pore densities of reactors, and the reaction rate (maximum of 

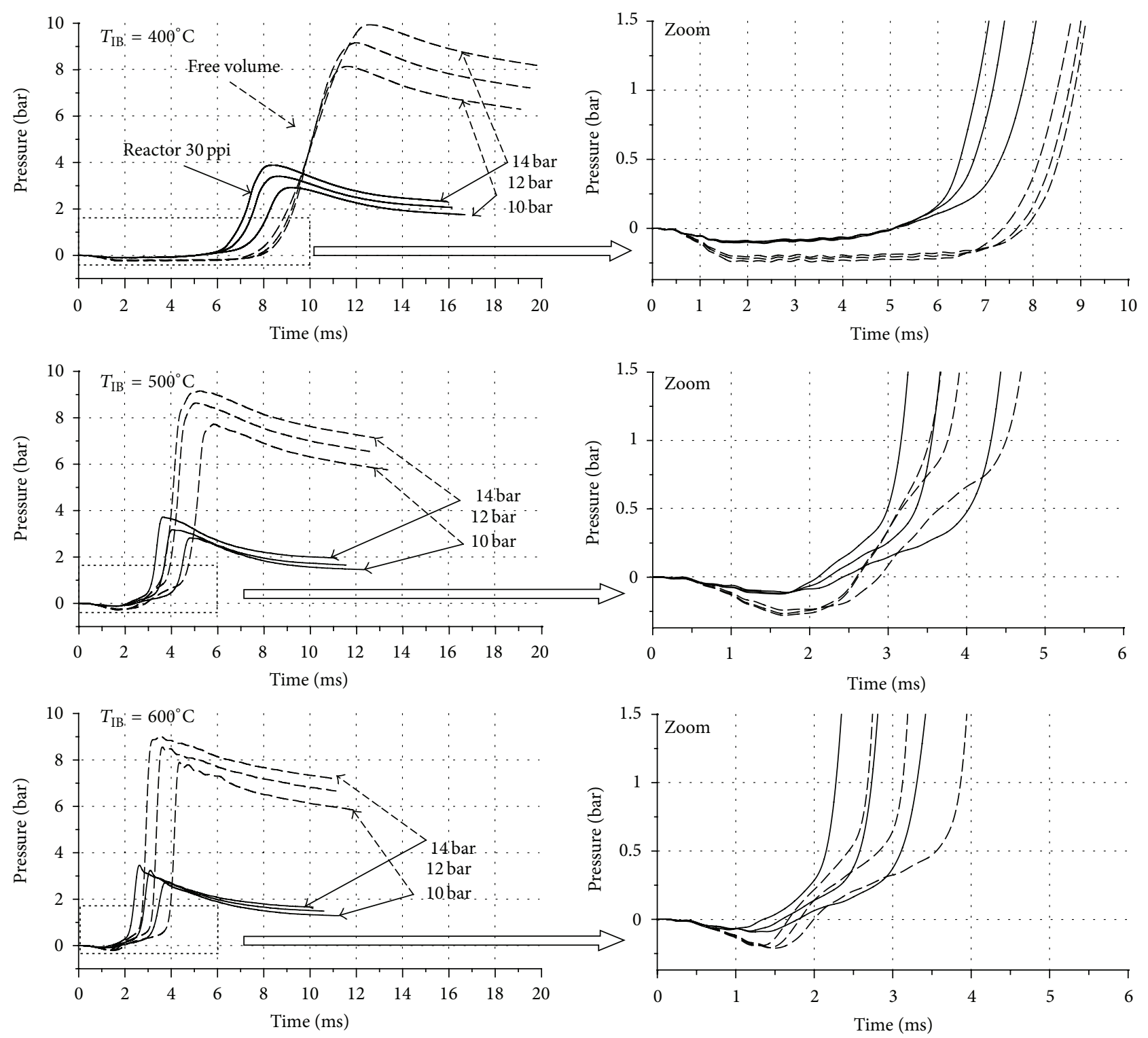

Figure 9: Comparison of pressure histories for heat release in free volume and in an $\mathrm{SiC}$ foam porous reactor (30 ppi) after fuel injection starts at three different initial temperatures $400^{\circ} \mathrm{C}, 500^{\circ} \mathrm{C}$, and $600^{\circ} \mathrm{C}$, and for three initial pressures $p_{\mathrm{IB}}=10 \mathrm{bar}, 12 \mathrm{bar}$ and $14 \mathrm{bar}$. The amount of injected fuel is $23.7 \mathrm{mg}$ : left—complete pressure history; right—initial period of the process limited to $1.5 \mathrm{bar}$.

peak) is clearly dependent on initial temperature. The level of the gradient peak is almost independent of the pore density at a given temperature. The qualitative shape of pressure and pressure gradient distributions is less pore density dependent and mainly depends on the initial temperature $T_{\mathrm{IB}}$.

At higher initial chamber pressures $\left(p_{\mathrm{IB}}=16 \mathrm{bar}\right)$, the influence of initial reactor temperature is even more visible (Figure 11). At $T_{\mathrm{IB}}=400^{\circ} \mathrm{C}$, the pressure curves show significantly different delay times for reactors with different pore densities, while at $T_{\mathrm{IB}}=600^{\circ} \mathrm{C}$ delay time is almost similar for all reactors investigated. The pressure peak generally decreases with increasing pore density at both initial temperatures, except with low density foams ( 8 and $10 \mathrm{ppi}$ ) that show quite similar peak levels. Initial temperature $T_{\mathrm{IB}}$ exerts some influence on the characteristic shape of pressure and pressure gradient histories, while reactor density shows no influence. At low initial temperature, the oxidation process in all reactors show a single-step distribution, while at high initial temperature the reaction is characterized by a multistep distribution. This observation is based on the investigation of pressure gradient distribution. In the case of higher temperature, this distribution shows a multimodal character indicating a multistep heat release process. At lower temperature, this behaviour is not clearly recognizable. The amount of fuel injected corresponds to the amount of energy supplied to the system (part of this energy is converted into heat). The effect of the amount of energy supplied to the system on the pressure histories after the start of fuel injection may be analyzed according to Figure 12 where the data are plotted for three different amounts of fuel at 

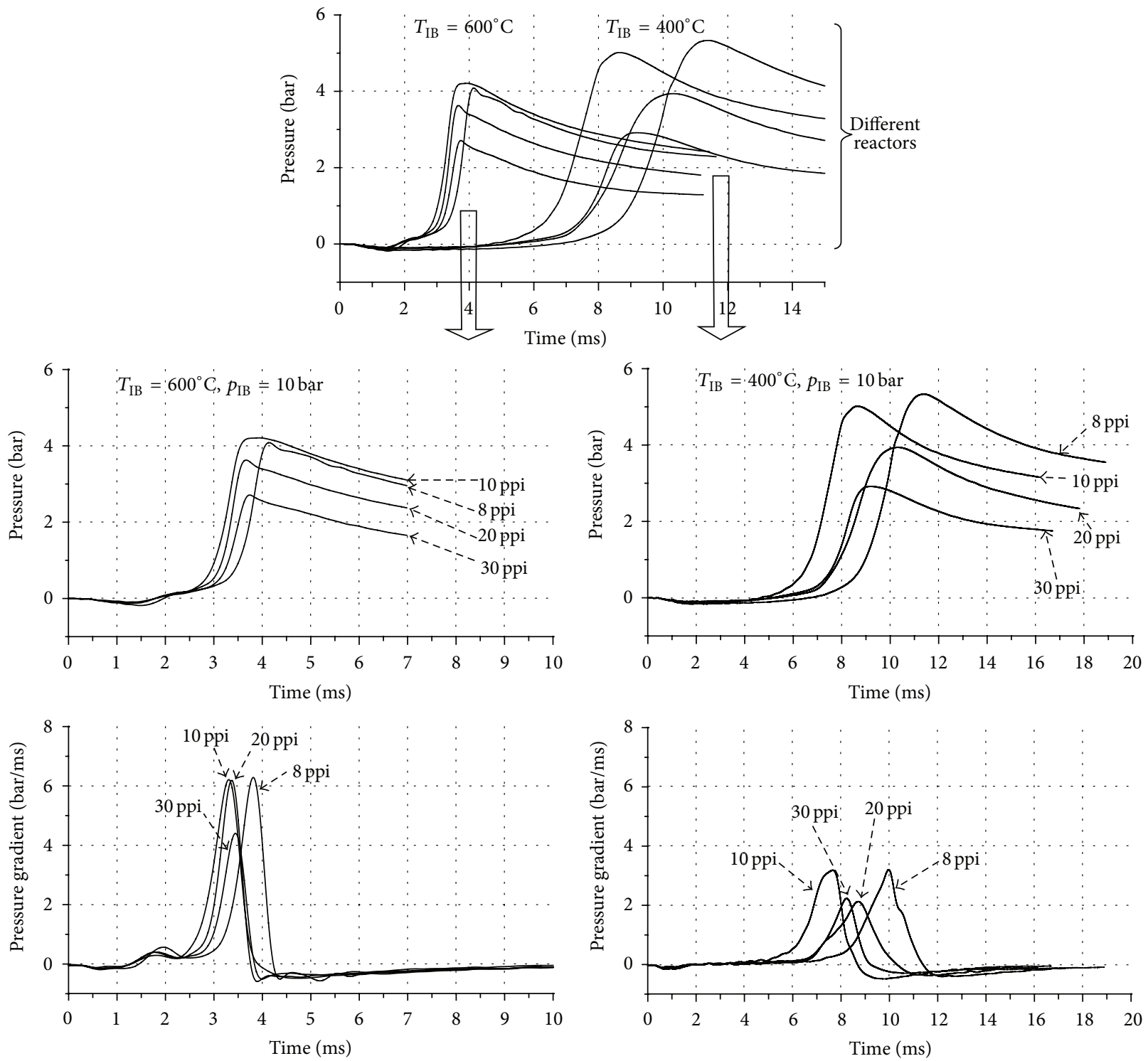

FIGURE 10: Comparison of pressure histories and pressure gradient distributions for heat release in different $\mathrm{SiC}$ foam porous reactors after fuel injection starts at two initial reactor temperatures $400^{\circ} \mathrm{C}$ (right) and $600^{\circ} \mathrm{C}$ (left) and for initial pressure $p_{\mathrm{IB}}=10$ bar. The amount of injected fuel is $23.7 \mathrm{mg}$.

constant air excess ratio resulting in variable initial pressure and initial temperature conditions. For a small amount of energy supplied $(7.5 \mathrm{mg})$ at low initial pressure and reactor temperature $T_{\mathrm{PM}}=400^{\circ} \mathrm{C}$, only small changes in the chamber pressure are recorded (as compared to other cases having more energy supplied); the amount of released heat is small, and a relatively large amount of this heat is accumulated in the porous reactor. With increasing mass of injected fuel, more heat is released faster within the combustion chamber, which results in more significant changes in the temperature of the gas trapped in the reactor volume. Increasing amount of supplied heat and initial temperature accelerate the process and increase the heat release rate. The role of reactor pore density in influencing heat capacity and heat transfer properties is clearly indicated in this figure. More energy accumulated in the reactor with higher pore density results in reduced pressure peaks under all investigated conditions. The heat release process dependence on initial reactor temperature and pressure for a constant air excess ratio for different investigated foam reactors is illustrated in Figure 13. Independent of the reactor properties, the heat release process is accelerated with increasing reactor temperature and initial pressure. The maximum gas temperature in a porous reactor (calculated from the pressure history of the heat release process at constant volume) significantly depends on the amount of heat accumulated in the solid phase of the reactor (see Figure 14). For all reactors, maximum combustion temperature is significantly reduced as compared to the free volume combustion chamber. For reactors with higher pore density, the maximum temperature is significantly reduced 

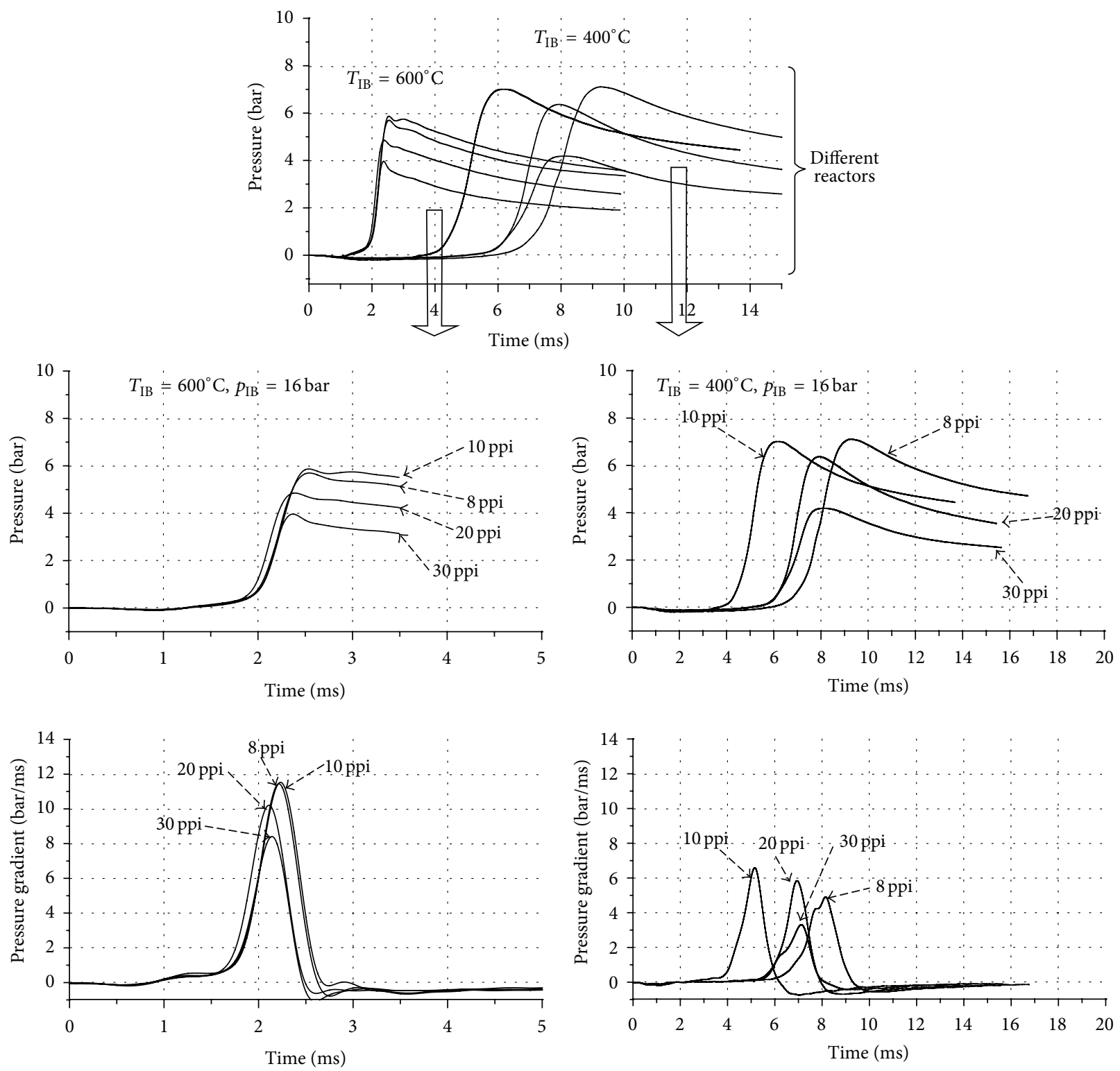

FIGURE 11: Comparison of pressure histories and pressure gradient distributions for heat release in different SiC foam porous reactors after fuel injection starts at two initial reactor temperatures $400^{\circ} \mathrm{C}$ (left) and $600^{\circ} \mathrm{C}$ (right) and for initial pressure $p_{\mathrm{IB}}=16$ bar. The amount of injected fuel is $23.7 \mathrm{mg}$.

at all initial pressures. This is especially visible in a reactor made of wire packing of significantly higher heat capacity as compared to foam reactors (see Table 1).

Almost constant temperature recorded in porous reactors independent of initial pressure indicates the role of heat capacity of reactor and heat transfer conditions. Both define the amount of heat accumulated in the porous reactors. Such an effect has been observed at all initial temperatures investigated. The maximum combustion temperature, however, gradually increases with initial chamber temperature, this temperature being lower in value with combustion reactors as with free volume systems. Again, reactors of higher heat capacity and higher pore density have lower gas temperature. The energy accumulated in a porous reactor, however, influences the oxidation process dynamics. Distribution of ignition delay time is plotted in Figure 15 versus initial chamber pressure for different porous reactors. Data are analysed for three initial reactor temperatures $T_{\mathrm{PM}}=400^{\circ} \mathrm{C}$, $500^{\circ} \mathrm{C}$, and $600^{\circ} \mathrm{C}$. For comparison, the plots for free Diesel injection are also presented in this figure. Generally, the delay time decreases with increasing initial chamber pressure $p_{\mathrm{IB}}$ and initial chamber temperature. The combustion process as performed in a porous reactor shows qualitative behavior similar to that in a free volume, but the delay time is reduced, especially at lower initial temperatures. Similarly to free volume combustion, the delay times at higher reactor temperatures are similar and much longer at $400^{\circ} \mathrm{C}$. At even lower initial temperatures, the process in a free volume system indicates the existence of a pressure range characterized by the shortest delay time and the highest 


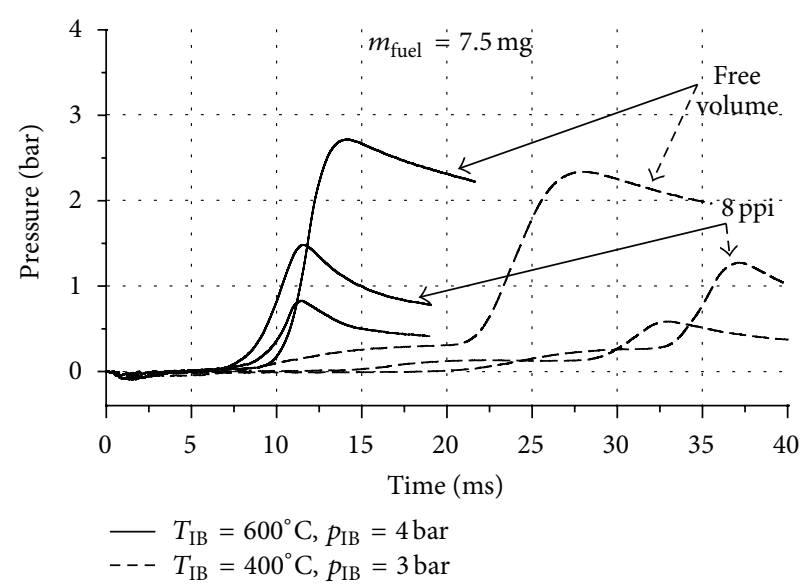

(a)

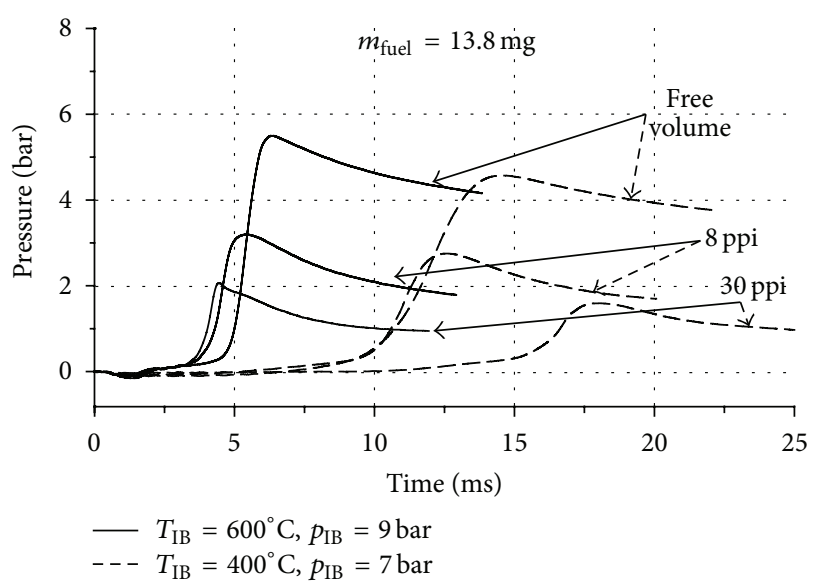

(b)

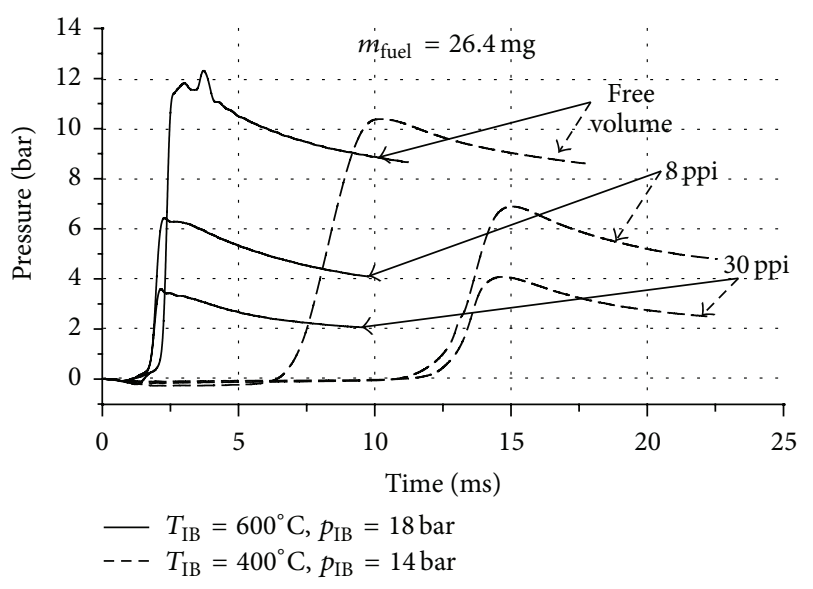

(c)

FIgURE 12: Comparison of pressure histories for heat release in different SiC foam porous reactors and in free volume combustion chamber after fuel injection starts for different mass of injected fuel at different initial pressure for two initial reactor temperatures $\left(400^{\circ} \mathrm{C}-\mathrm{dashed}\right.$ line; $600^{\circ} \mathrm{C}$-solid line).

reaction rate. The authors have defined this pressure region as a positive pressure coefficient (PPC) analogous to the negative temperature coefficient, as described in [7].

Reaction rate represented by pressure gradients after the begin of fuel injection depends significantly on the porous reactor's heat capacity and pore density as compared to the free volume process (Figure 16). Generally, the reaction rate increases with increasing initial chamber pressure and with increasing initial temperature, but the latter dependence may be reactor specific. Higher initial reactor temperature means more energy accumulated in the reactor and less influence of the gas temperature on the reaction rate. The maximum combustion temperature increases with increasing initial chamber pressure and results in increasing reaction rate. For reactors having large heat capacity and small pores (high density foam reactor and wire packing reactor), the heat release rate is lowered due to the large amount of heat transferred to the reactor. It must be noted that different pore densities and pore structures (especially for reactors with small pores and high density, for example, wire packing reactors) give different conditions for fuel injection into the reactor. This results in different mixture formation conditions. These conditions, however, are very complex and it is still very difficult to describe them [3-5].

4.4. Overall Characteristics of the Heat Release Process. Summarizing their process analysis for free volume and individual reactors, the authors constructed fields representing characteristic combustion modes in porous reactors as compared to free volume Diesel injection conditions. These fields consider two kinds of parameters characterizing lowand high-temperature oxidation processes: distribution of characteristic reaction behavior represented by a single- and multistep oxidation (Figure 17) and distribution of characteristic delay time (Figure 18). All these data are plotted in a two-dimensional field of initial pressure and temperature. The data have been grouped according to preselected criteria, such as number of slopes in the reaction curve and duration of delay time. The data plotted have quantitative form, and only the shape of marked border lines among different combustion modes should be interpreted more qualitatively. There are three characteristic regions selected in Figure 17 representing three different characteristic modes of the oxidation process. 


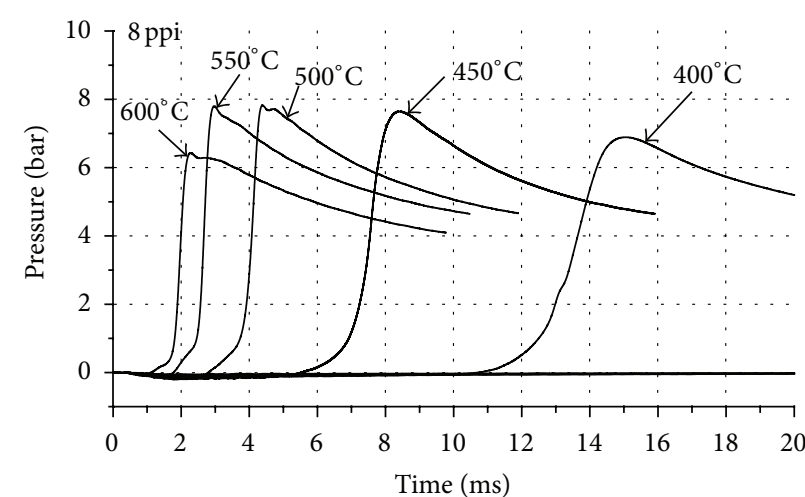

(a)

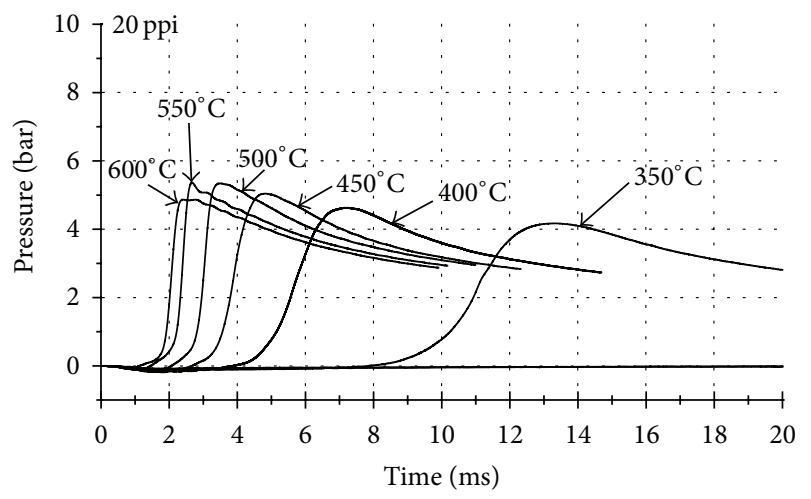

(c)

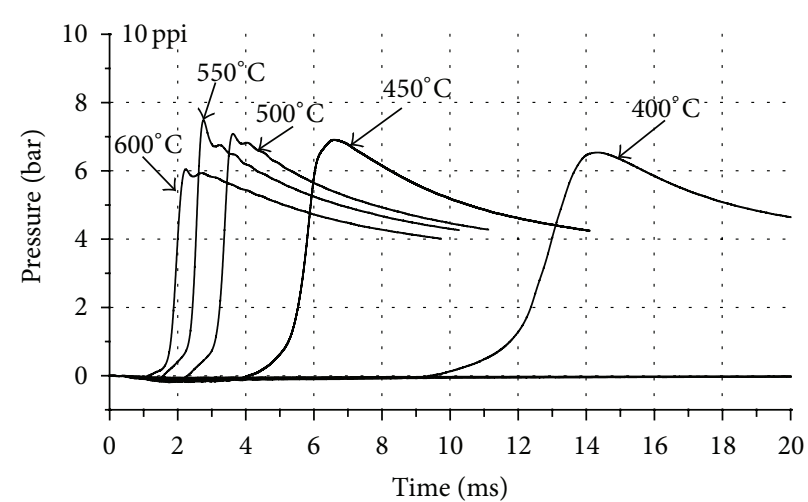

(b)

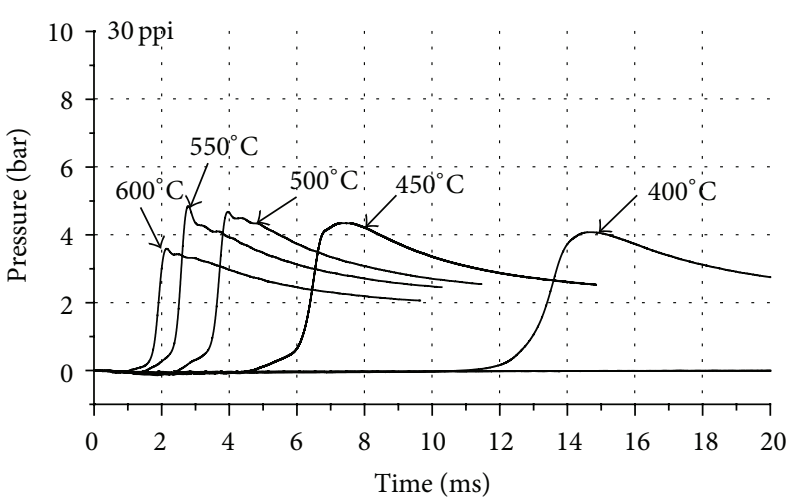

(d)

FIGURE 13: Comparison of pressure histories for heat release in different SiC foam porous reactors after fuel injection starts for a constant mass of injected fuel $(26.3 \mathrm{mg}$ ) and for a constant air excess ratio (initial pressure and initial temperature are not constant).

(i) Region 1 is characterized by single-step reactions and is located at lower initial temperatures for all initial pressures. The shape of the border line depends on the reactor heat capacity, pore density, and pore structure.

(ii) Region 2 is characterized by multistep reactions with two slopes recognizable in the reaction curve and is located in the range of middle-high initial temperatures at middle-high initial pressures. The shape of border line depends on the reactor heat capacity, pore density, and pore structure.

(iii) Region 3 is characterized by multistep reactions with three slopes recognizable in the reaction curve and is located in the range of higher initial temperatures at low-to-middle initial pressures. The shape of the border line depends on the reactor heat capacity, pore density, and pore structure.

In the case of reaction delay time, the following regions have been selected in Figure 18.

(i) Region A is characterized by very long delay times $t>20 \mathrm{~ms}$ and is located at lower initial temperatures at all initial pressures investigated. The shape of the border line depends on the reactor heat capacity, pore density, and pore structure. (ii) Region B is characterized by delay times $10 \mathrm{~ms}<t \leq$ $20 \mathrm{~ms}$ and is located at higher initial temperatures and lower initial pressures as well as in a small region of high initial pressures. The shape of the border line depends on the reactor heat capacity, pore density, and pore structure.

(iii) Region $\mathrm{C}$ is characterized by delay times $5 \mathrm{~ms}<t \leq$ $10 \mathrm{~ms}$ and is located at higher initial temperatures and lower-to-middle initial pressures as well as in a small region of high initial pressures. The shape of the border line depends on the reactor heat capacity, pore density, and pore structure.

(iv) Region $\mathrm{D}$ is characterized by delay times $2 \mathrm{~ms}<t \leq$ $5 \mathrm{~ms}$ and is located at higher initial temperatures and middle-to-high initial pressures. The shape of the border line depends on the reactor heat capacity, pore density, and pore structure.

(v) Region $\mathrm{E}$ is characterized by very short delay times $t \leq 2 \mathrm{~ms}$ and is located at high initial temperatures and high initial pressures. The shape of the border line depends on the reactor heat capacity, pore density, and pore structure.

Analysis of characteristic regions selected in Figures 17 and 18 indicates qualitative similarity of heat release processes as performed under Diesel-like and in porous 


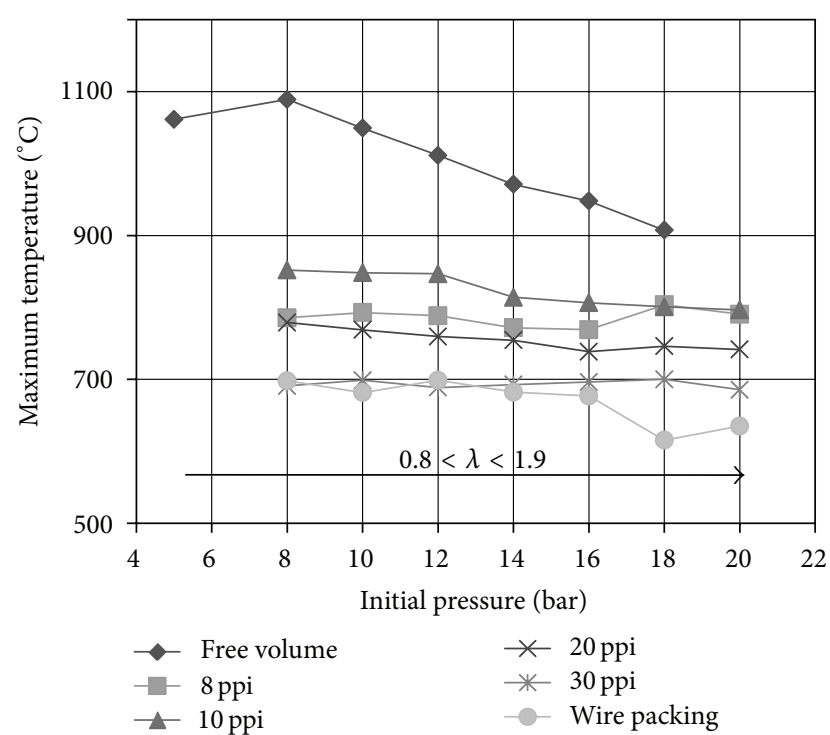

FIGURE 14: Comparison of maximum combustion temperature for heat release in free volume and in different porous reactors $(\mathrm{SiC}$ foam reactors and steel wire packing reactor) as a function of initial pressure $p_{\text {ІВ }}$ for a constant mass of injected fuel $(23.7 \mathrm{mg})$.

reactor conditions. A quantitative influence of porous-reactor features (reactor heat capacity, pore density, pore structure, specific surface area and fuel distribution in the reactor volume) is visible, especially if comparing the free volume system with high-density reactors. This qualitative similarity of processes as performed in a free volume and in porous reactors indicates a high probability for applicability of the concept with clean combustion in a porous reactor to internal combustion engine conditions. Quantitative influence of reactor conditions described by reactor heat capacity, pore density, pore structure, specific surface area, fuel distribution in the reactor volume, and heat transport indicates a large potential for system optimization for application to internal combustion engines.

In the case of engine-like conditions with direct fuel injection into reactor, there is a very complex process concerning spray interaction with porous structure, homogenization in reactor volume, heat transfer, and fuel vaporization. The present results show that the oxidation processes in porous reactor, may be accelerated as compared to the free volume chamber under reduced maximum temperature conditions. For proper mixture formation and combustion condition in porous reactor a nearly homogeneous charge simultaneously ignited in reactor volume is required, but all oxidation processes must be completed not locally in a single pore, but globally in a large volume of the reactor. This, in combination with heat accumulation in the reactor structure, permits completeness of the combustion process at significantly reduced temperature (comparing to the temperature peaks in a free combustion volume system). In a real porous reactor with direct fuel injection process, mixture formation process is much more homogeneous with significantly improved vaporization degree. Additionally, the "combustion chamber" consists of porous reactor with nearly constant thermodynamic conditions in the whole reactor volume (temperature) allowing completion of oxidation process (e.g., $\mathrm{CO}, \mathrm{HC}$ ). The heat accumulated in the reactor also extends the lean limit of the process, as compared to free volume combustion.

\section{Concluding Remarks}

Heat release processes in porous reactors have been analyzed under Diesel engine-like conditions as simulated in a special combustion chamber with a common-rail Diesel injection system. For comparison, the heat release in a free volume combustion chamber has also been analyzed. In all systems investigated, the fuel was directly injected into the combustion chamber, and the processes of mixture formation in porous reactors and in free volume chambers have turned out to be different. The heat release process has been investigated over a wide range of initial pressures and temperatures simulating thermodynamic conditions in an engine at the moment when fuel injection starts. Pressure history after the begin of fuel injection in porous reactors and in free volume chambers significantly depends on the initial pressure and temperature. At lower initial temperatures, the process is accelerated in porous reactors. Combustion in porous reactors is characterized by additional heat accumulation in the reactor's solid phase as compared to a free volume chamber. This results in significantly reduced pressure peaks and lowered combustion temperature level. This may directly be used for controlling and reducing of thermal $\mathrm{NO}_{x}$ formation in a real engine. Reactor parameters such as heat capacity, pore density, specific surface area, pore structure, and heat transport properties significantly influence the mixture formation and heat release process. Process delay time is significantly dependent on initial pressure and initial temperature, and, on the whole, decreases with pressure and with increasing temperature. This behavior has been observed in a free volume chamber and in all porous reactors investigated. Heat release rate increases with initial pressure and initial temperature: in a porous reactor it significantly depends on the amount of heat accumulated in the reactor as a function of reactor heat capacity, pore density, specific surface area, and pore structure. Characteristic modes of the heat release process in a two-dimensional field of initial chamber pressure and temperature have been selected: both for a free volume combustion chamber and for different porous reactors. There are three characteristic regions representing the different character of the process represented by a single- and multistep oxidation. Region 1 is characterized by single-step reactions and is located at lower initial temperatures for all initial pressures. Region 2 is characterized by multistep reactions with two slopes recognizable in the reaction curve and is located in the range of middle-to-high initial temperatures at middle-to-high initial pressures. Region 3 is characterized by multistep reactions with three slopes recognizable in the reaction curve and is located in the range of higher initial temperatures at low-to-middle initial pressures. The shape of all border lines depends on the reactor's heat capacity, pore density, and pore structure. Analysis of characteristic 


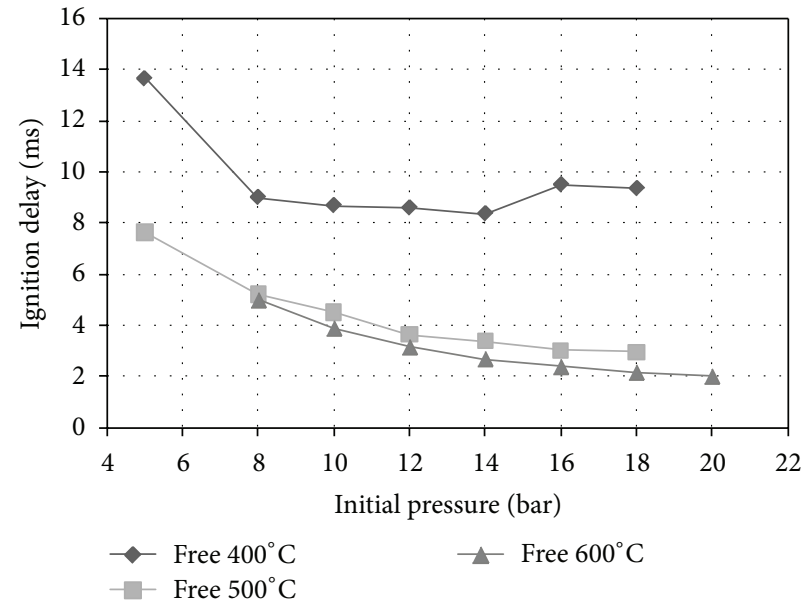

(a)

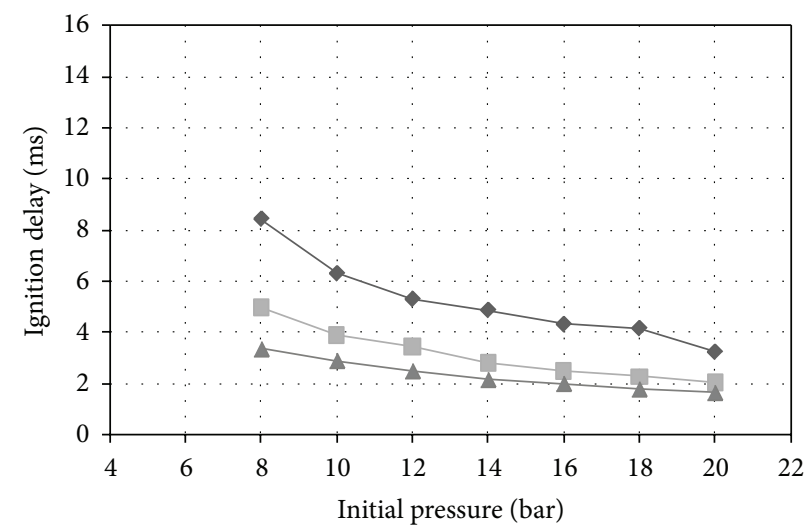

$\longrightarrow 10 \mathrm{ppi}-400^{\circ} \mathrm{C}$
$-\quad 10 \mathrm{ppi}-500^{\circ} \mathrm{C}$

-10 ppi- $600^{\circ} \mathrm{C}$

(c)

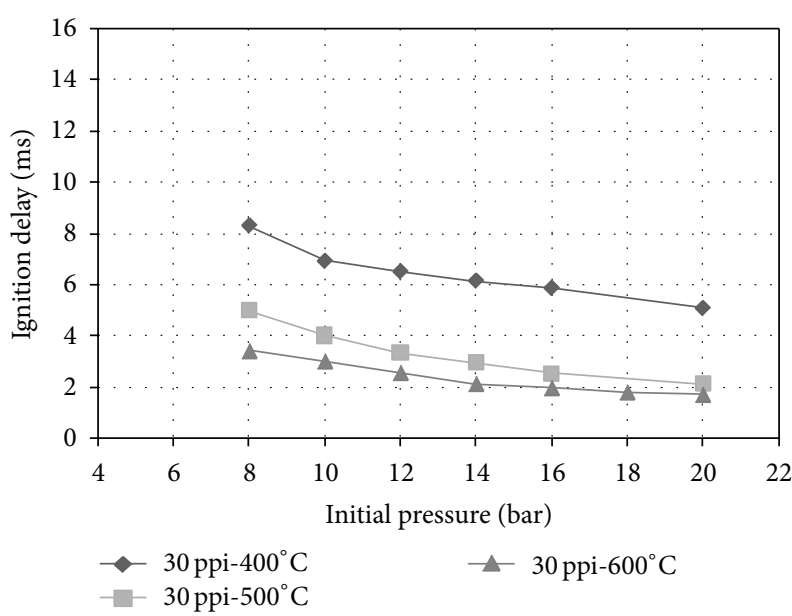

(e)

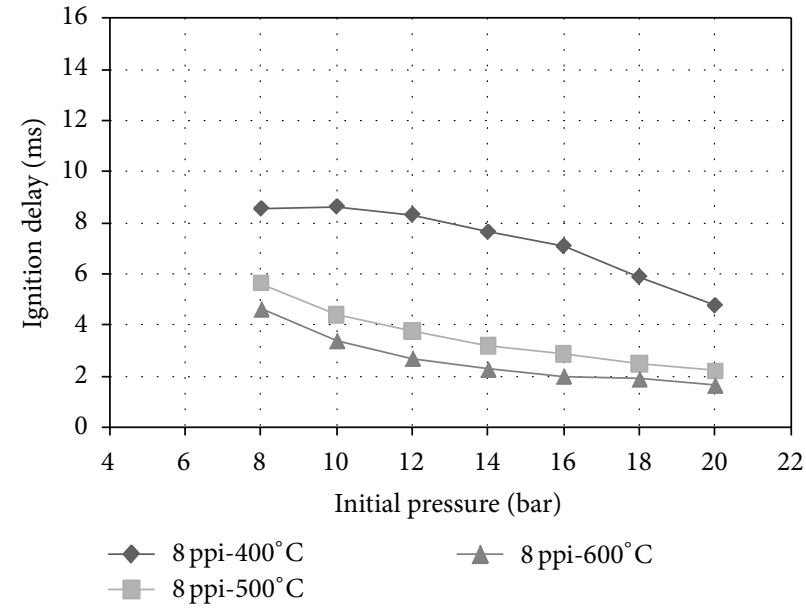

(b)

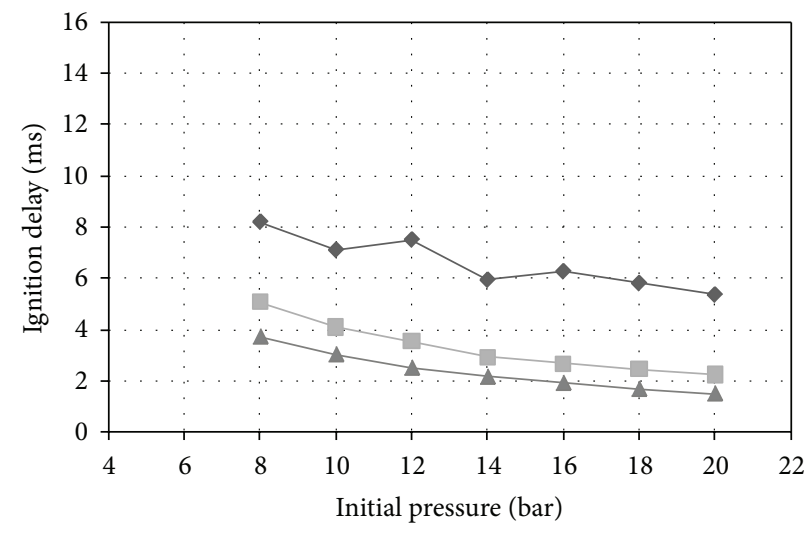

$\begin{array}{ll}\longrightarrow & 20 \mathrm{ppi}-400^{\circ} \mathrm{C} \\ - & -20 \mathrm{ppi}-500^{\circ} \mathrm{C}\end{array}$

(d)

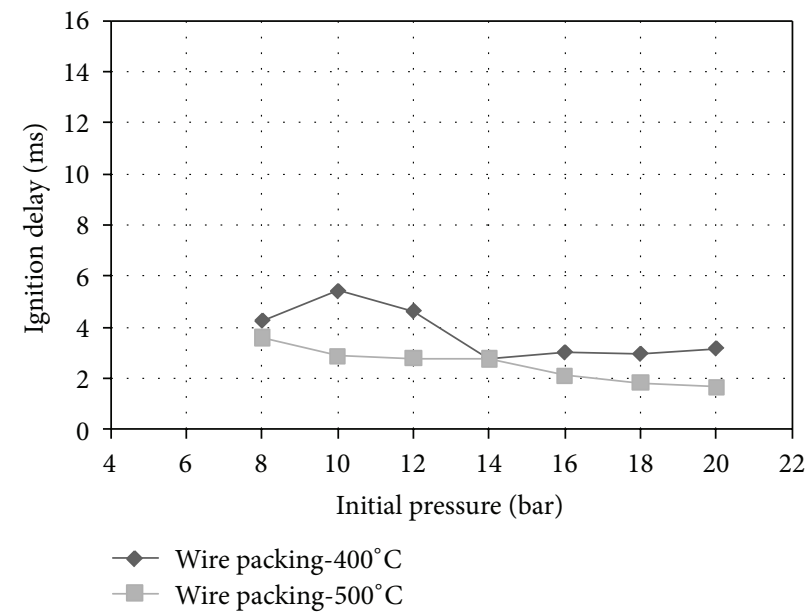

(f)

FIGURE 15: Comparison of delay time for heat release in free volume and in different porous reactors (SiC foam reactors and steel wire packing reactor) as a function of initial pressure $p_{\mathrm{IB}}$ for three inital temperatures $400^{\circ} \mathrm{C}, 500^{\circ} \mathrm{C}$, and $600^{\circ} \mathrm{C}$ (mass of injected fuel is $23.7 \mathrm{mg}$ ). 


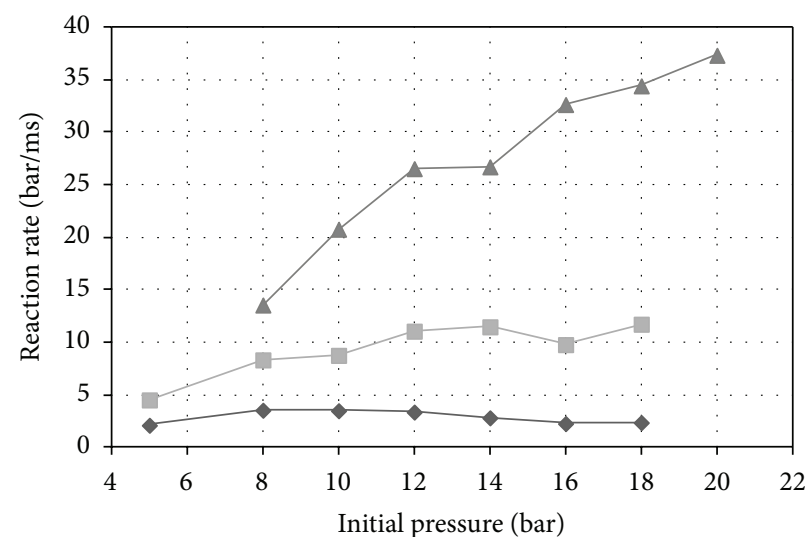

$\rightarrow$ Free $400^{\circ} \mathrm{C}$

$\triangle$ Free $600^{\circ} \mathrm{C}$

(a)

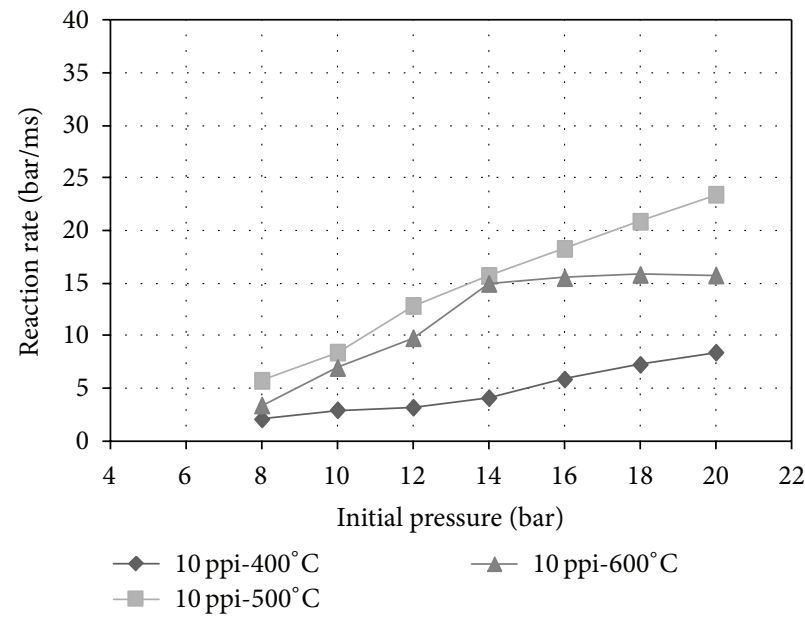

(c)

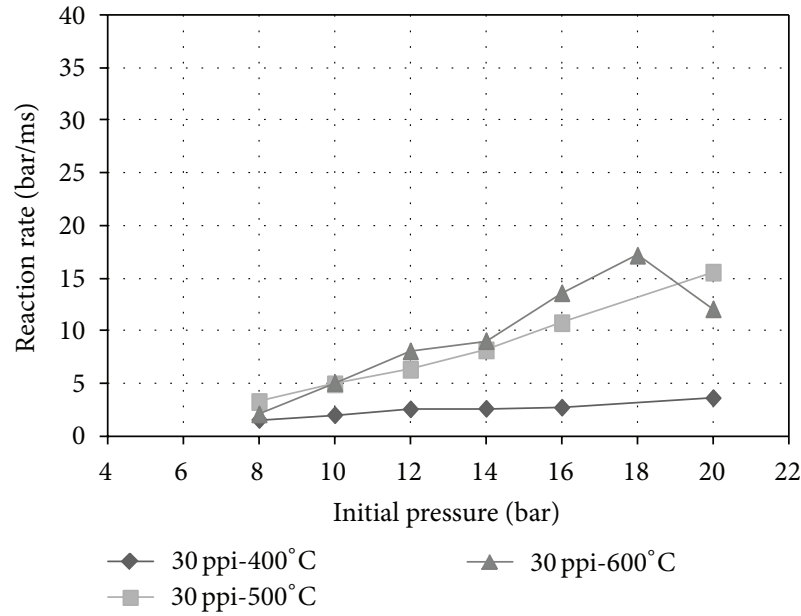

(e)

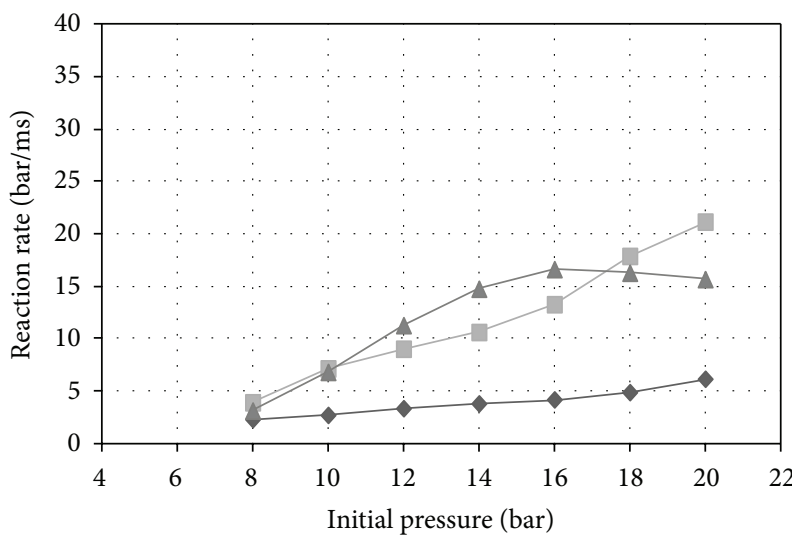

- 8 ppi $-400^{\circ} \mathrm{C}$

$-8 \mathrm{ppi}-500^{\circ} \mathrm{C}$

$\neg 8$ ppi $-600^{\circ} \mathrm{C}$

(b)

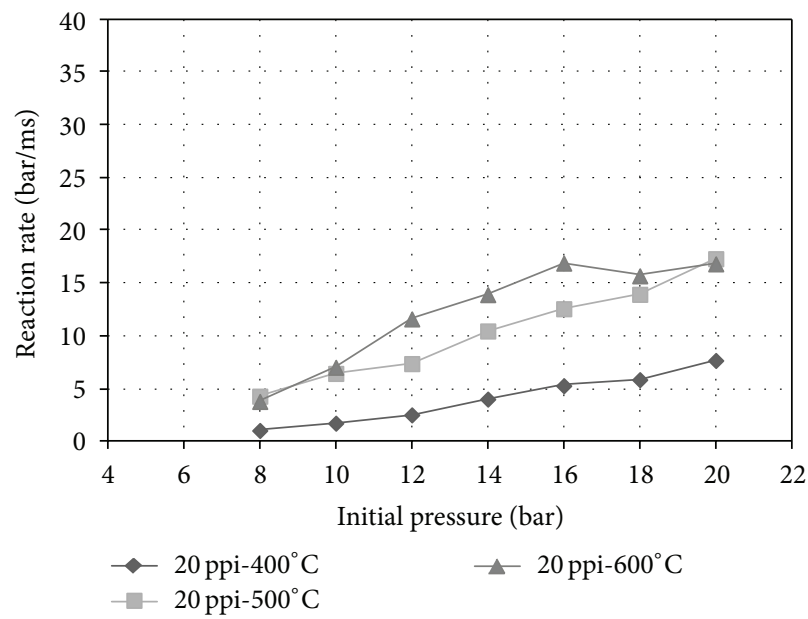

(d)

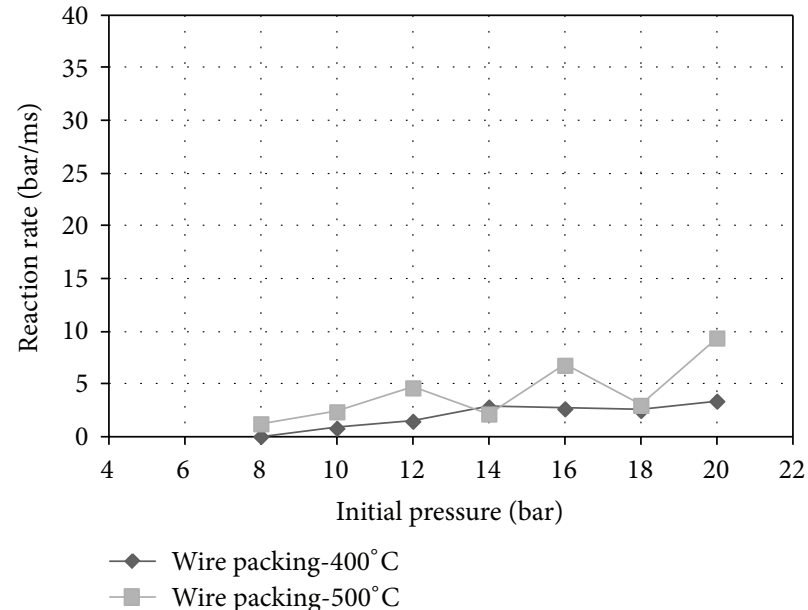

(f)

FIGURE 16: Comparison of pressure gradient (reaction rate) for heat release in free volume and in different porous reactors (SiC foam reactors and steel wire packing reactor) as a function of initial pressure $p_{\mathrm{IB}}$ for three initial temperatures $400^{\circ} \mathrm{C}, 500^{\circ} \mathrm{C}$, and $600^{\circ} \mathrm{C}$ (mass of injected fuel is $23.7 \mathrm{mg}$ ). 


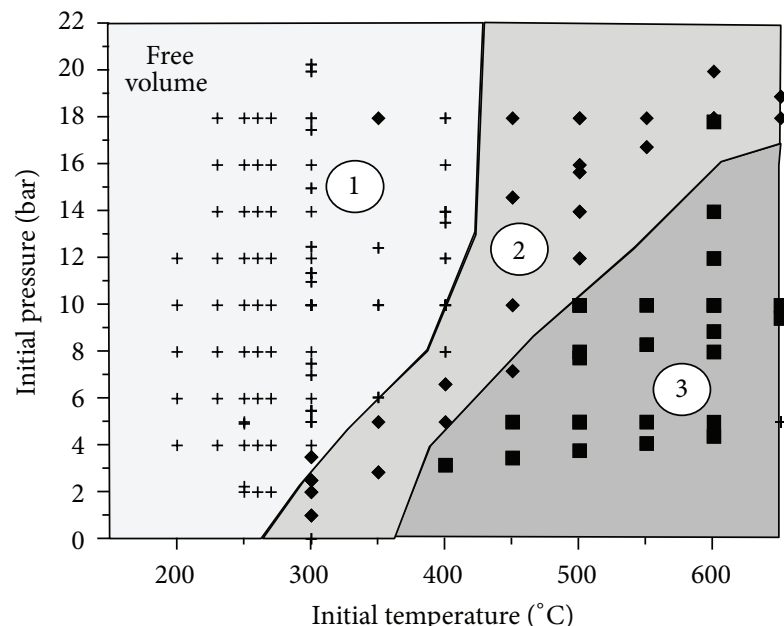

(a)

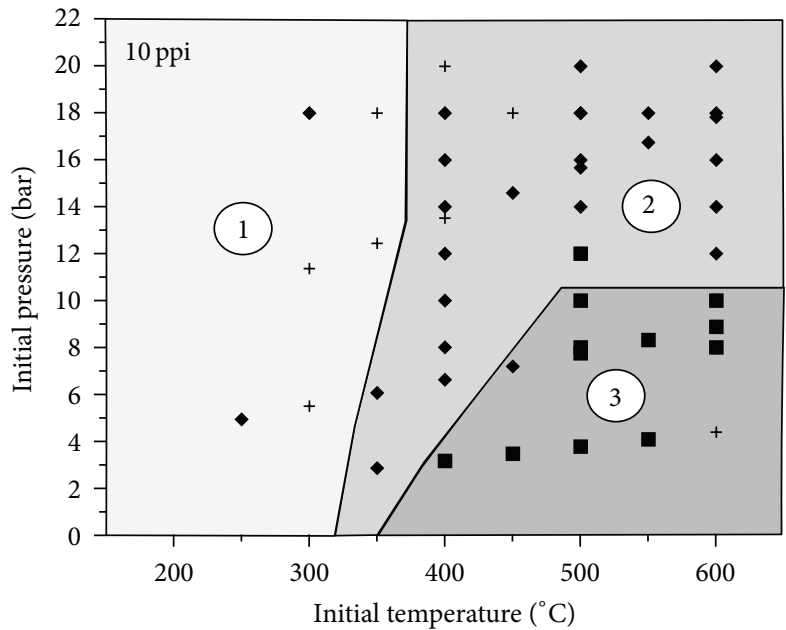

(c)

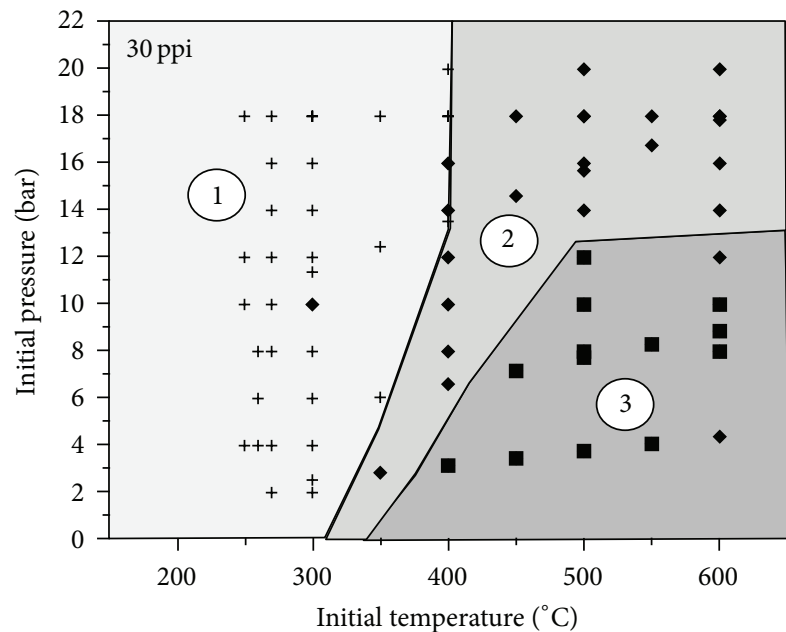

+ Single-step reaction (1 slope)

- Multistep reaction (2 slopes)

- Multistep reaction (3 slopes)

(e)

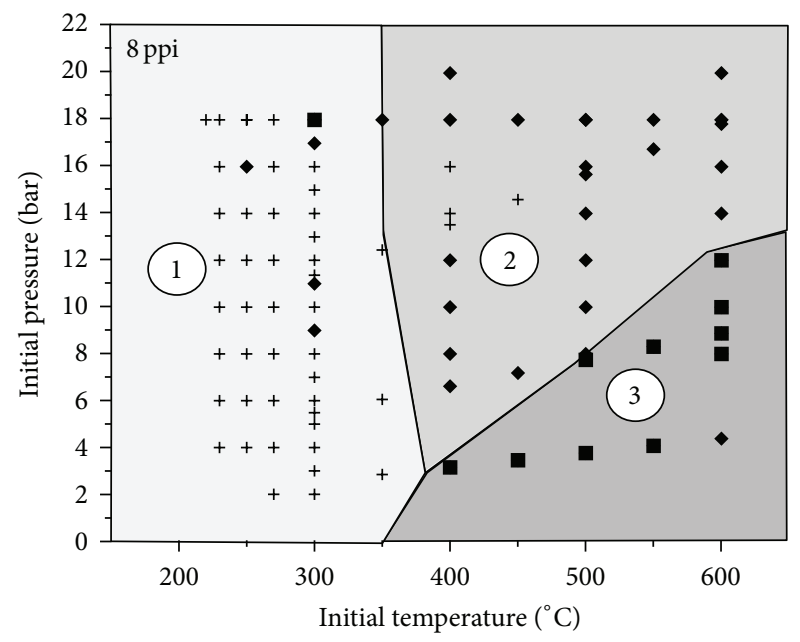

(b)

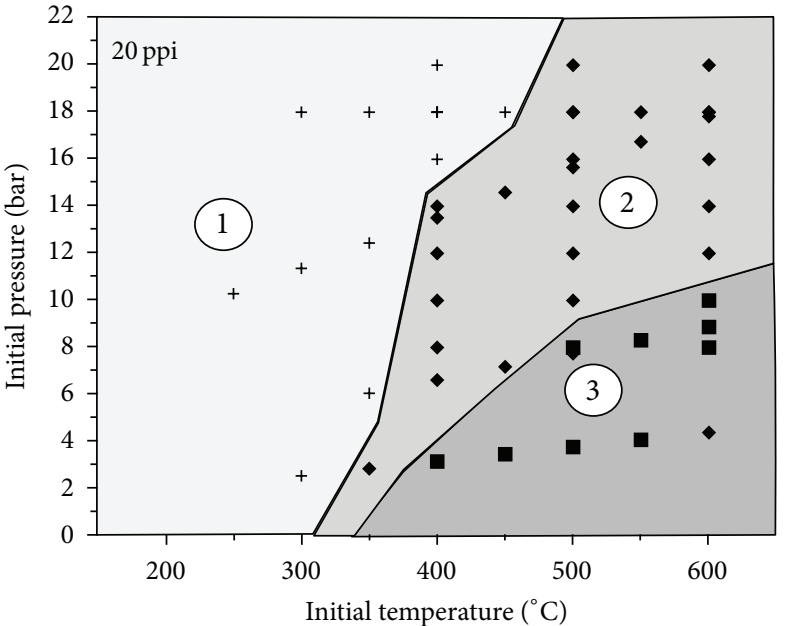

(d)

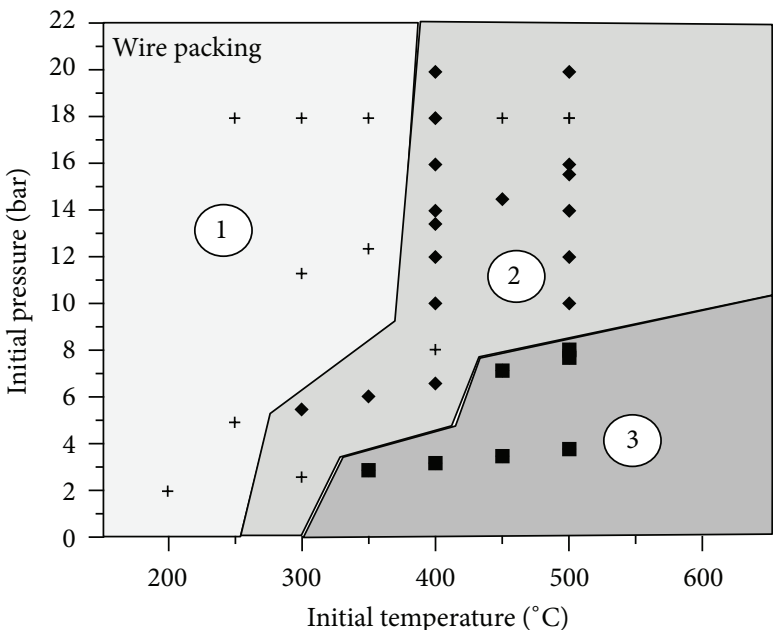

+ Single-step reaction (1 slope)

- Multistep reaction (2 slopes)

- Multistep reaction (3 slopes)

(f)

FIGURE 17: Fields representing characteristic combustion modes in porous reactors as compared to free Diesel injection conditions. 


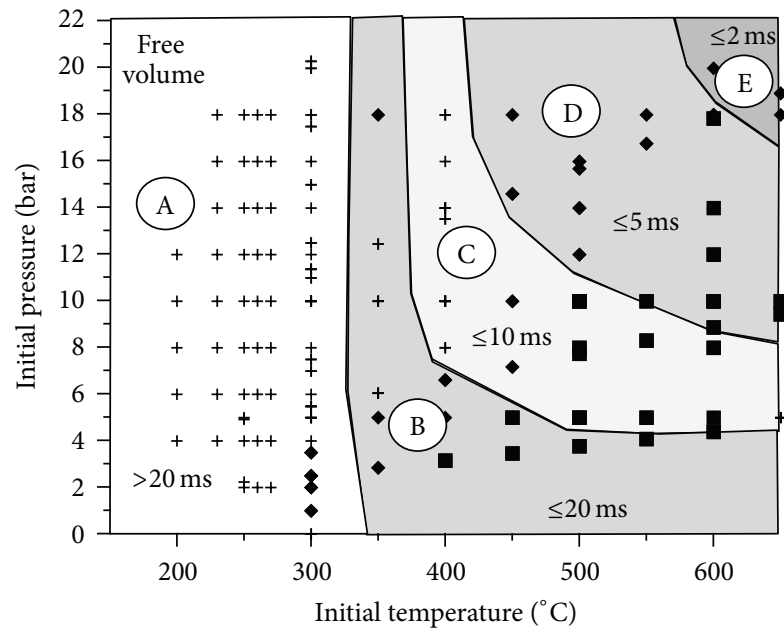

(a)

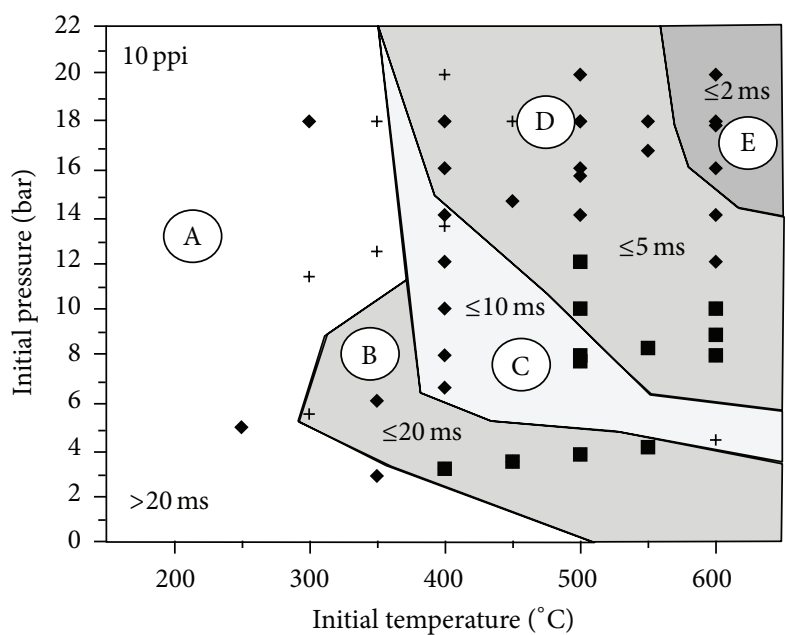

(c)

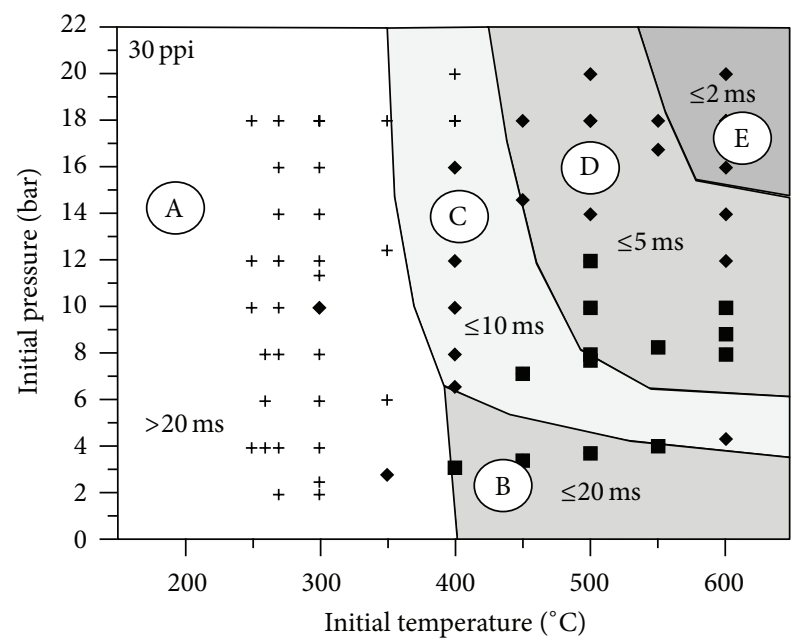

+ Single-step reaction (1 slope)

- Multistep reaction (2 slopes)

- Multistep reaction (3 slopes)

(e)

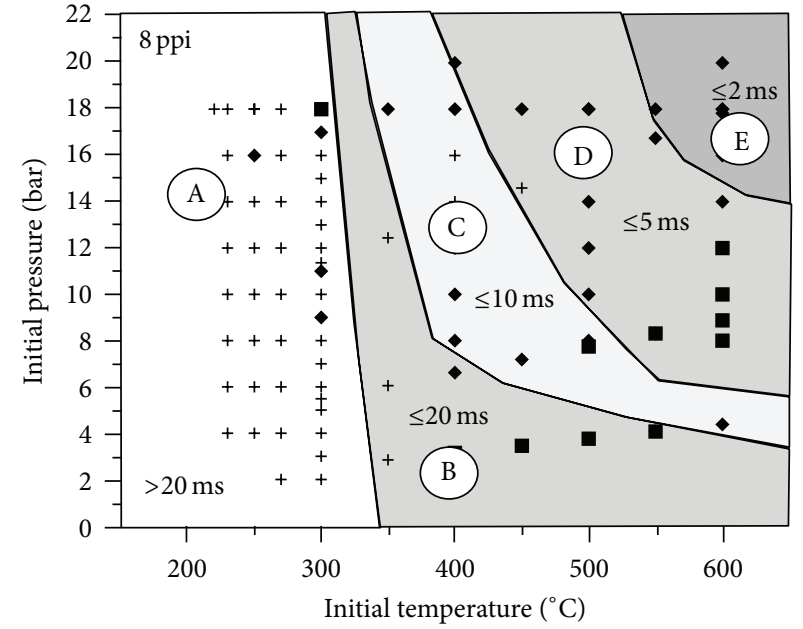

(b)

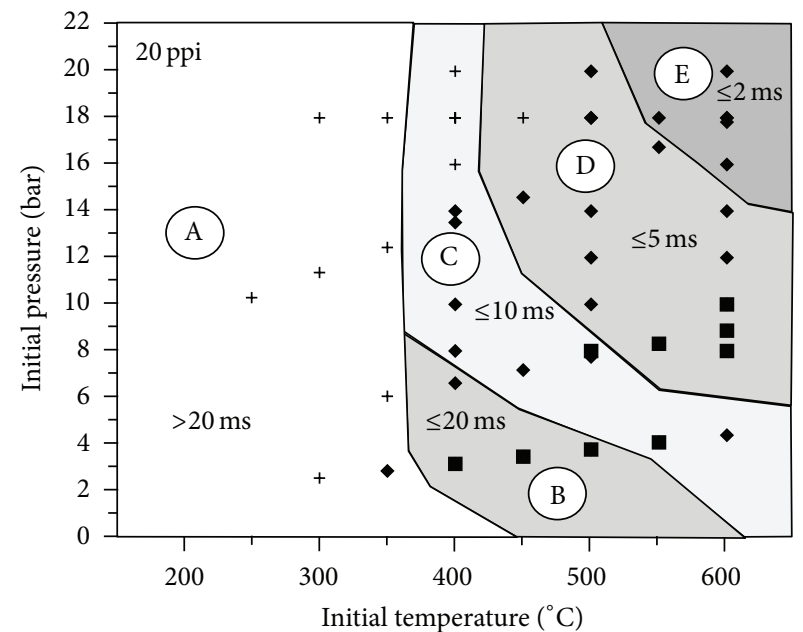

(d)

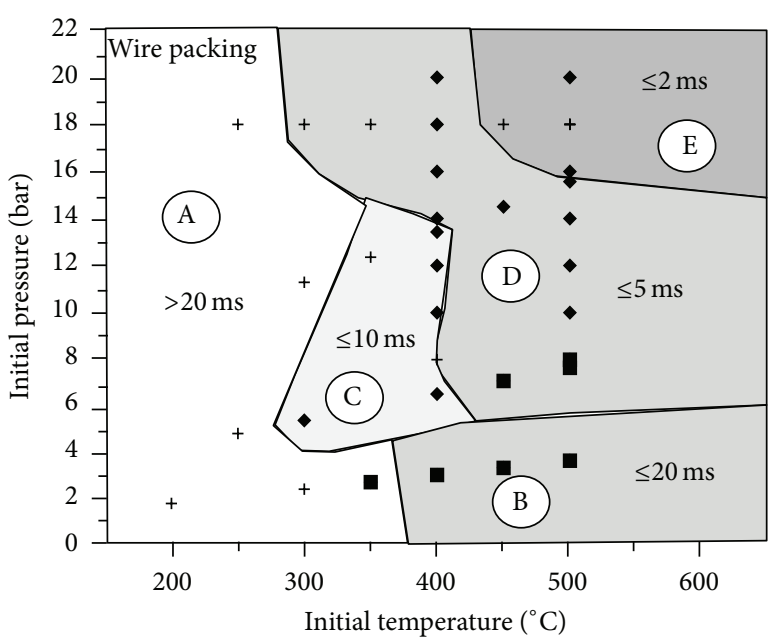

+ Single-step reaction (1 slope)

- Multistep reaction (2 slopes)

- Multistep reaction (3 slopes)

(f)

FIGURE 18: Fields representing characteristic combustion modes and delay times in porous reactors as compared to free Diesel injection conditions. 
delay time distribution in a two-dimensional field of initial chamber pressure and temperature allowed selection of five characteristic ranges. Region $\mathrm{A}$ is characterized by delay times $t>20 \mathrm{~ms}$ and is located at lower initial temperatures at all initial pressures investigated. Region B is characterized by delay times $10 \mathrm{~ms}<t \leq 20 \mathrm{~ms}$ and is located at higher initial temperatures and lower initial pressures as well as in a small region of high initial pressures. Region $\mathrm{C}$ is characterized by delay times $5 \mathrm{~ms}<t \leq 10 \mathrm{~ms}$ and is located at higher initial temperatures and lower-to-middle initial pressures as well as in a small region of high initial pressures. Region $\mathrm{D}$ is characterized by delay times $2 \mathrm{~ms}<t \leq 5 \mathrm{~ms}$ and is located at higher initial temperatures and middleto-high initial pressures. Region $\mathrm{E}$ is characterized by delay times $t \leq 2 \mathrm{~ms}$ and is located at high initial temperatures and high initial pressures. The shape of all border lines depends on the reactor's heat capacity, pore density, and pore structure. Qualitative similarity of characteristic modes of the heat release process in a free volume and in porous reactors as performed under Diesel engine-like conditions indicates high probability of applicability of the combustion porous reactors to an internal clean combustion process. A quantitative influence of porous reactor features (heat capacity, pore density, pore structure, specific surface area, and fuel distribution in reactor volume) has been clearly indicated.

\section{Notations}

\begin{tabular}{|c|c|}
\hline$E_{\mathrm{ch}}:$ & $\begin{array}{l}\text { Amount of energy supplied with } \\
\text { injected fuel }[\mathrm{J}]\end{array}$ \\
\hline HCCI: & $\begin{array}{l}\text { Homogeneous charge compression } \\
\text { ignition }\end{array}$ \\
\hline$H_{u}:$ & Fuel heating value $[\mathrm{J} / \mathrm{kg}]$ \\
\hline IB: & Zero-time point (fuel injection start) \\
\hline$m_{\text {fuel }}:$ & Mass of injected fuel [mg] \\
\hline$p_{\mathrm{IB}}:$ & $\begin{array}{l}\text { Initial chamber pressure at the time } \\
\text { instance of fuel injection start [bar] }\end{array}$ \\
\hline PM: & Porous medium \\
\hline ppi: & Pore density (pores per linear inch) \\
\hline$T_{\mathrm{IB}}:$ & $\begin{array}{l}\text { Initial chamber temperature at the time } \\
\text { instance of fuel injection starts }\left[{ }^{\circ} \mathrm{C}\right]\end{array}$ \\
\hline$T_{\mathrm{PM}}:$ & $\begin{array}{l}\text { Porous reactor temperature at the time } \\
\text { instance of fuel injection starts }\left[{ }^{\circ} \mathrm{C}\right]\end{array}$ \\
\hline$T_{\max }:$ & Maximum combustion temperature \\
\hline$t:$ & $\begin{array}{l}\text { Characteristic (delay) time of reaction } \\
{[\mathrm{ms}]}\end{array}$ \\
\hline$Q_{\text {in }}:$ & Amount of heat released \\
\hline$\Delta p_{\mathrm{GAS}(\mathrm{FV})}$ & $\begin{array}{l}\text { Increase of gas pressure as a result of } \\
\text { heat release process in free volume } \\
\text { combustion chamber }\end{array}$ \\
\hline$\Delta p_{\mathrm{GAS}(\mathrm{PM})}:$ & $\begin{array}{l}\text { Increase of gas pressure as a result of } \\
\text { heat release process in porous reactor }\end{array}$ \\
\hline$\Delta T_{\mathrm{GAS}(\mathrm{FV})}$ & $\begin{array}{l}\text { Increase of gas temperature as a result } \\
\text { of heat release process in free volume } \\
\text { combustion chamber }\end{array}$ \\
\hline$\Delta U_{\mathrm{GAS}(\mathrm{FV})}:$ & $\begin{array}{l}\text { Increase of gas internal energy as a } \\
\text { result of heat release process in free } \\
\text { volume combustion chamber }\end{array}$ \\
\hline
\end{tabular}
$\Delta U_{\mathrm{GAS}}$ in PM: Increase of gas internal energy as a result of heat release process in porous reactor
$\Delta U_{\mathrm{PM}}: \quad$ Increase of reactor internal energy as a result of heat release process in porous reactor
$\lambda:$ Air excess ratio

\section{Acknowledgments}

M. Weclas thanks the Federal Ministry of Education and Research (BMBF) and German Federation of Industrial Research Associations (AiF) for financial support of the presented investigation (Project no. 17N2207). The authors thank Mr. B. Leykauf and T. Plecher for their support in performing of the investigation presented.

\section{References}

[1] F. Durst and M. Weclas, "A new type of internal combustion engine based on the porous-medium combustion technique," Journal of Automobile Engineering, IMechE, part D, vol. 215, no. 1, pp. 63-81, 2001.

[2] J. Cypris, L. Schlier, N. Travitzky, P. Greil, and M. Weclas, "Heat release process in three-dimensional macro-cellular $\mathrm{SiC}$ reactor under Diesel engine-like conditions," Fuel, vol. 102, pp. 115-128, 2012.

[3] M. Weclas, "Some fundamental observations on the diesel jet destruction and spatial distribution in highly porous structures," Journal of Porous Media, vol. 11, no. 2, pp. 125-144, 2008.

[4] M. Weclas and J. Cypris, "Characterization of distribution nozzle operation for mixture homogenization by late diesel injection strategy," Journal of Automobile Engineering, IMechE, part D, vol. 226, pp. 529-546, 2012.

[5] M. Weclas, J. Cypris, and T. M. A. Maksoud, "Diesel spray interaction with highly porous structures for supporting of liquid distribution in space and its vaporization," in Proceedings of the 4th International Conference on Porous Media and its Applications in Science and Engineering (ICPM4 '12), Potsdam, Germany, 2012.

[6] N. Shahangian and J. Ghojel, "Investigation of the interaction between diesel spray and porous medium," in Proceedings of the 17th Australasian Fluid Mechanics Conference, Auckland, New Zealand, December 2010.

[7] M. Weclas, "Characterization of low- and high-temperature oxidation pro-cesses under non-premixed Diesel-engine like conditions," International Journal of Engine Research, vol. 13, no. 6, pp. 628-638, 2012.

[8] S. Tanaka, F. Ayala, J. C. Keck, and J. B. Heywood, “Two-stage ignition in HCCI combustion and HCCI control by fuels and additives," Combustion and Flame, vol. 132, no. 1-2, pp. 219-239, 2003.

[9] P. Cheng and H. Zhu, "Effects of radial thermal dispersion on fully-developed forced convection in cylindrical packed tubes," International Journal of Heat and Mass Transfer, vol. 30, no. 11, pp. 2373-2383, 1987.

[10] A. A. Mohamad, S. Ramadhyani, and R. Viskanta, "Modelling of combustion and heat transfer in a packed bed with embedded coolant tubes," International Journal of Heat and Mass Transfer, vol. 37, no. 8, pp. 1181-1191, 1994.

[11] A. A. M. Oliveira and M. Kaviany, "Nonequilibrium in the transport of heat and reactants in combustion in porous media," 
Progress in Energy and Combustion Science, vol. 27, no. 5, pp. 523-545, 2001.

[12] A. Mohammadi, M. Ziabasharhagh, and A. Jazayeri, "Numerical simulation of porous medium internal combustion engine," in Proceedings of ASME-JSME-KSME Joint Fluids Engineering Conference (AJK2011-FED '11), Hamamatsu, Japan, July 2011, paper no. AJK2011-03079.

[13] M. Weclas, "Potential of porous media combustion technology as applied to internal combustion engines," Journal of Thermodynamics, vol. 2010, Article ID 789262, 39 pages, 2010.

[14] G. A. Merkel, T. Tao, and W. A. Cutler, "New cordierite diesel particulate filters for catalyzed and non-catalyzed applications," in Proceedings of the 6th International Congress on Catalysis and Automotive Pollution Control, 2003.

[15] W. A. Cutler, "Overview of ceramic materials for diesel particulate applications," in Proceedings of the 28th International Cocoa Beach Conference on Advanced Ceramics \& Composites, 2004.

[16] F. Ruiz, "Regenerative internal combustion engine. Part I. Theory," Journal of Propulsion and Power, vol. 6, no. 2, pp. 203208, 1990.

[17] F. Ruiz, "Regenerative internal combustion engine. Part II. Practical configurations," Journal of Propulsion and Power, vol. 6, no. 2, pp. 209-213, 1990.

[18] S. Thyageswaran and F. Ruiz, "Time-dependent analysis of the regenerative engine cycle," SAE Technical Paper 900912, 1990.

[19] A. J. Ferrenberg, "Low heat rejection regenerated enginesa superior alternative to turbocompounding," SAE Technical Paper 940946, 1994.

[20] A. Ferrenberg and B. E. Williams, "Progress in the development of the regenerated diesel engine," SAE Technical Paper 961677, 1996.

[21] M. Kaviany, "In cylinder-thermal regeneration: porous-foam engine regenerator," in Principles of Heat Transfer in Porous Media, Springer, New York, NY, USA, 1999.

[22] C.-W. Park and M. Kaviany, "Evaporation-combustion affected by in-cylinder, reciprocating porous regenerator," Journal of Heat Transfer, vol. 124, no. 1, pp. 184-194, 2002.

[23] K. Hanamura and S. Nishio, "A feasibility study of reciprocatingflow super-adiabatic combustion engine," in Proceedings of the 6th ASME-JSME Thermal Engineering Joint Conference, 2003, Paper No. TED-AJ03-547.

[24] H. Liu, M. Xie, and D. Wu, "Simulation of a porous medium (PM) engine using a two-zone combustion model," Applied Thermal Engineering, vol. 29, no. 14-15, pp. 3189-3197, 2009.

[25] M. A. Mujeebu, M. Z. Abdullah, A. A. Mohamad, and M. Z. A. Bakar, "Trends in modeling of porous media combustion," Progress in Energy and Combustion Science, vol. 36, no. 6, pp. 627-650, 2010.

[26] M. Weclas, J. Cypris, and M. A. Maksoud, “Thermodynamic properties of real porous combustion reactor under Diesel engine-like conditions," Journal of Thermodynamics, vol. 2012, Article ID 798104, 11 pages, 2012. 

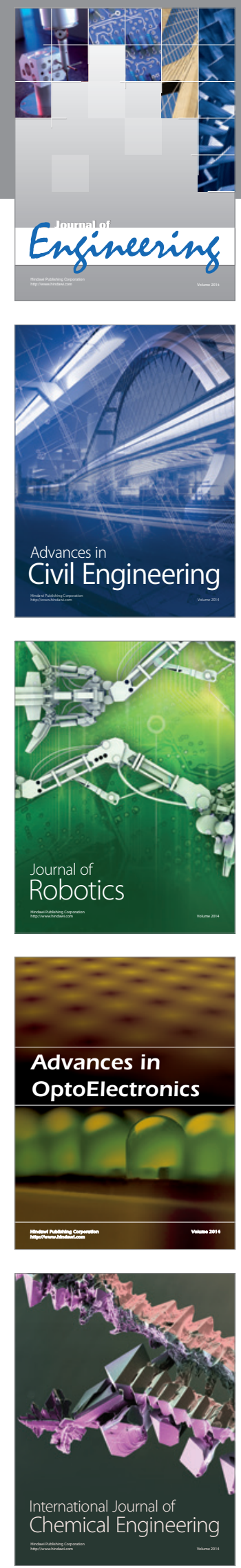

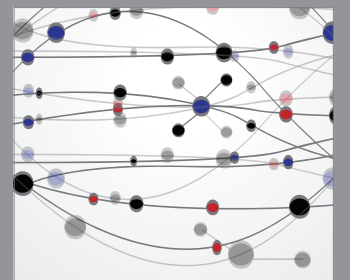

The Scientific World Journal
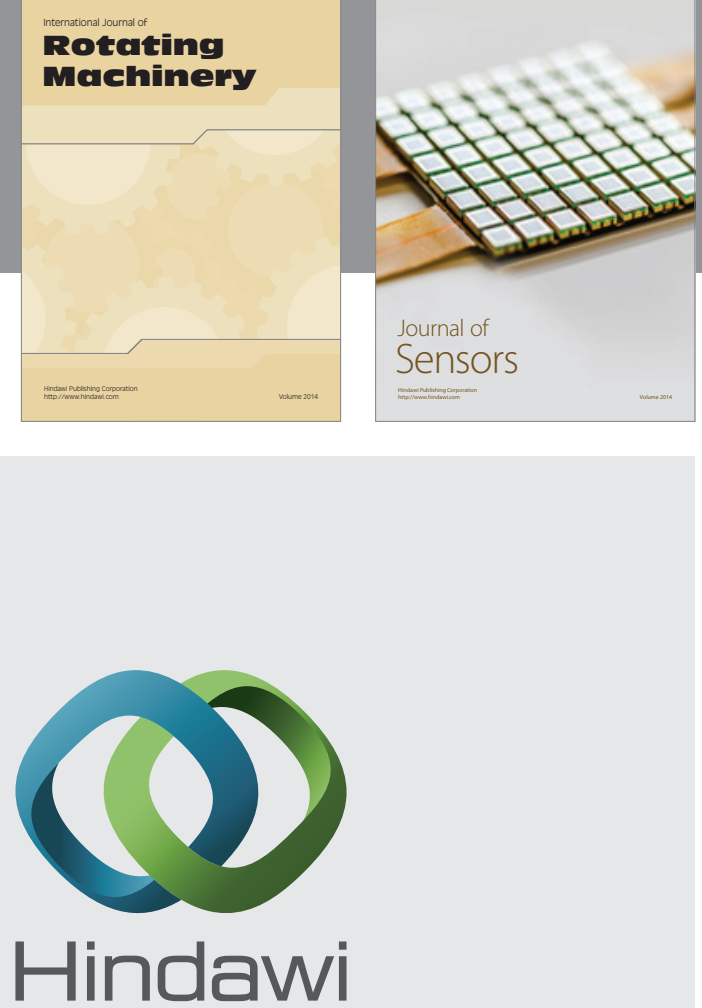

Submit your manuscripts at http://www.hindawi.com
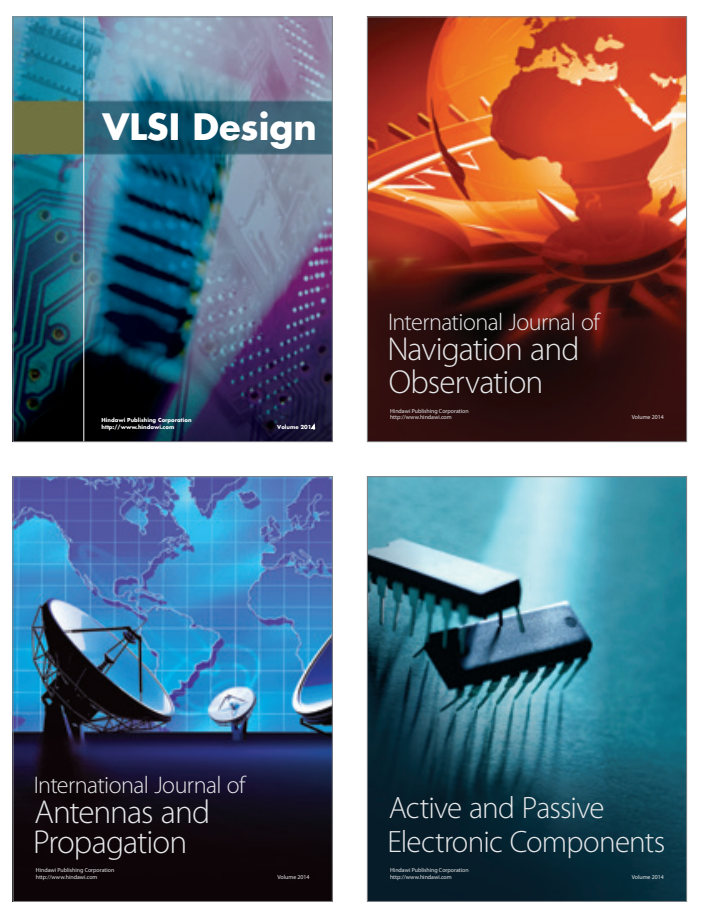
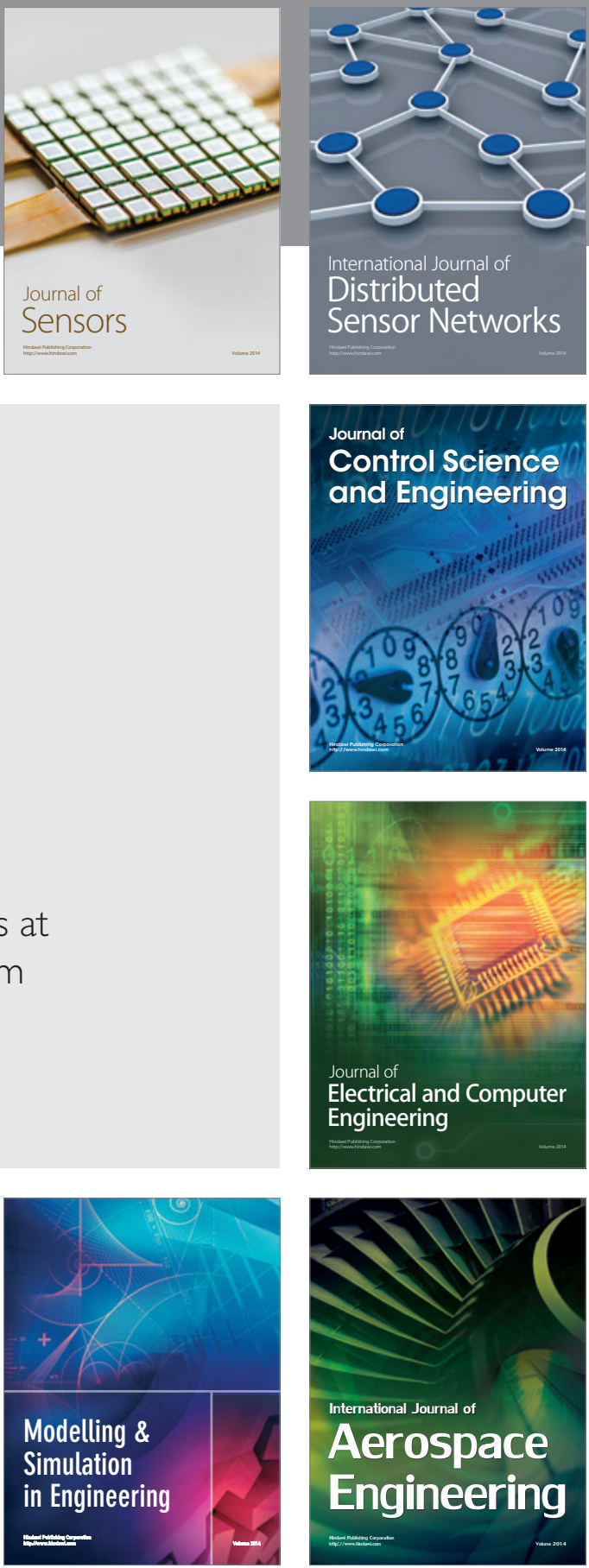

Journal of

Control Science

and Engineering
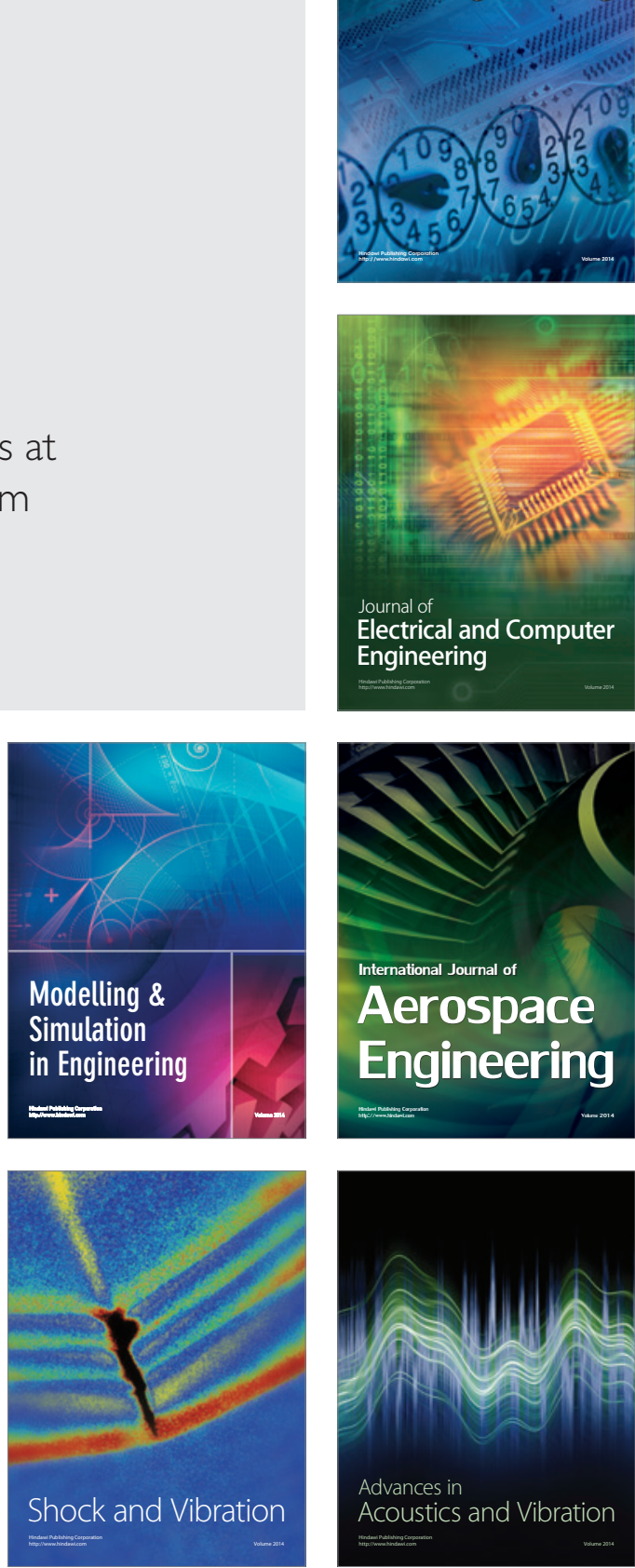